\title{
Effect of feed flow pattern on the distribution of permeate fluxes in desalination by direct contact membrane distillation
}

\author{
Sofiane Soukane ${ }^{1}$, Wahib M. Naceur ${ }^{2}$, Lijo Francis ${ }^{3}$, Ahmad Alsaadi $^{3}$, Noreddine Ghaffour ${ }^{3}$ \\ ${ }^{1}$ Institute of Marine Science and Coastal Management, Campus Universitaire de Dely Ibrahim, Bois des Cars, \\ BP 19, 16320, Algiers, Algeria \\ ${ }^{2}$ University of Blida 1, Process Engineering Department, BP 270 Blida, Algeria \\ ${ }^{3}$ King Abdullah University of Science and Technology (KAUST), Water Desalination and Reuse Center \\ (WDRC), Division of Biological \& Environmental Science \& Engineering (BESE), 23955-6900 Thuwal 23955- \\ 6900, Saudi Arabia, Tel. +966-128082180,Email: noreddine.ghaffour@kaust.edu.sa
}

\begin{abstract}
The current study aims to highlight the effect of flow pattern on the variations of permeate fluxes over the membrane surface during desalination in a direct contact membrane distillation (DCMD) flat module. To do so, a three dimensional (3D) Computational Fluid Dynamics (CFD) model with embedded pore scale calculations is implemented to predict flow, heat and mass transfer in the DCMD module. Model validation is carried out in terms of average permeate fluxes with experimental data of seawater desalination using two commercially available PTFE membranes. Average permeate fluxes agree within $6 \%$ and less with experimental values without fitting parameters. Simulation results show that the distribution of permeate fluxes and seawater salinity over the membrane surface are strongly dependent on momentum and heat transport and that temperature and concentration polarization follow closely the flow distribution. The analysis reveals a drastic effect of recirculation loops and dead zones on module performance and recommendations to improve MD flat module design are drawn consequently.
\end{abstract}

Keywords: Membrane distillation; 3D CFD modeling; Permeate flux distribution; Salinity distribution; Polarization.

\section{Introduction}

Membrane distillation (MD), among the most promising separation techniques for its low cost operation, has achieved the prototyping stage. There is now a need for proper MD module design for efficient process integration and scale-up [1-5]. MD is a thermal process 
and it is now well established that flow rate and inlet temperatures have a major impact on resulting permeate fluxes, thus performance. However, as in any chemical process equipment, flow distribution in MD remains driven by module design, such as its length, feed/permeate channel height, fluid inlet and outlet location, as well as operating conditions including inlet temperatures and fluid flow rate. Computational Fluid Dynamics (CFD) codes, which are available in commercial or open source versions, are now used in MD to assess process and equipment performance [6-16]. These codes, when associated with powerful mesh generators and post-processors, solve coupled momentum, heat and species transport to provide critical information, including, fluid velocity, pressure, temperature and chemical species distribution in complex computational domains. This information can then be used judiciously to improve process design and efficiency while reducing costly experimental trials. Generally, the flow approach for full size equipment is similar in all research efforts with an imposed velocity at the inlet and no slip boundary condition at the domain walls. However, the difficulty in CFD modeling of MD often lies in the choice of the boundary condition for the heat transfer problem. The nonlinear heat transfer mechanism across the membrane represents the major hurdle in DCMD analysis with contribution from both conduction and mass transfer. The difficulty in the calculation of the surface temperature at both sides of the membrane pushed researchers to tackle the task as a conjugate heat transfer problem by including the permeate side in the computational domain. However, commercial codes do not always offer easy implementation options to account for mass transfer contribution to heat transfer across the membrane. Early use of commercial CFD codes for DCMD was demonstrated by Katsandri and Vahdati [17], who performed 3D simulations of flat membrane module with spacers using ANSYS CFX. Yu et al. [18] used fluent 6.3 to investigate hollow fiber DCMD module by considering a constant mass transfer coefficient. Cipollina et al. [11] used the commercial software ANSYS CFX11.0 to simulate flow and heat transfer and assigned a constant heat flux at the domain boundaries. Similarly, Al-Sharif et al. [6] adopted a 3D approach in which they used OpenFOAM, an open source CFD code, and assigned a constant heat flux as a boundary condition for heat transfer. Shakaib et al. [8] used the commercial code FLUENT 6.3 and assigned a constant heat flux at the boundaries. Yu et al. [9] used FLUENT 6.3 assuming a constant membrane coefficient, which sets the rate of species transferred through the membrane thus the vaporization rate. Janajreh and Suwwan [19] presented a coupled approach taking into account both feed and permeate sides of the module. The authors update the temperature profiles after accounting for the latent heat of vaporization and re-run the 
flow model. However, the frequency of the temperature profile update is not mentioned and the authors present only two dimensional simulations. Later, the authors validated a conjugate approach on a flat DCMD module [20]. More recently, Katsandri [21] circumvented ANSYS CFX restriction by applying appropriate heat and mass transfer fluxes at the interface between feed and permeate domains. Chang et al. [22, 23] used fluent 6.3 to analyze flow in DCMD channels with and without spacers. The contribution of mass transport to heat transfer across the membrane was considered as source terms at both membrane sides. In a more recent contribution the authors investigated heat transfer coefficients in flat DCMD modules [24]. Hasanizadeh et al. [25] used COMSOL v3.4 to model a 2D representation of a flat DCMD module. However, their model does not include the contribution of vapor transport to overall heat transfer across the membrane. Further contributions can be found in a detailed review by Shirazi et al. [26] which reports the current state of the art of CFD modeling in MD. Interestingly, the authors state that although only temperature polarization is known to significantly affect the MD process, mass transfer should be included for an in-depth understanding of the underlying physical phenomena.

It is shown that, for the DCMD scale under investigation, it is possible to solve the coupled problem without the permeate side included in the computational domain while reaching desired accuracy and agreement with experiments. Indeed, the inclusion of the permeate side is often a way to circumvent the difficulty of computing the permeate side temperature of the membrane surface since the latter is left to the CFD code and becomes part of the solution of the conjugate problem. In the approach presented herein, fully coupled flow, heat and mass transfer problems are solved in the feed side of a laboratory scale size DCMD module using ANSYS-fluent [27], while the permeate side is replaced by an appropriate boundary condition. The boundary condition at the membrane for the heat transfer problem is of mixed type, thus depends on a heat transfer coefficient as well as the boundary temperature. The presence of the membrane is considered as a resistance to heat transfer and both contributions from conduction and vaporization due to species transport are taken into account. The heat transfer modes are known to depend on the local surface temperature at both sides of the membrane as well as salinity at the feed side. The boundary condition at the membrane surface for species transfer is of flux type, set by the value of the permeate flux. The temperature and salinity distribution on the feed side are given by the CFD calculations while the transport problem at the membrane scale is solved locally to determine the temperature on the permeate side and consequently the rate of distilled species. The in-house procedure used 
to solve non-linear transport at the pore scale is embedded in an in-house user routine provided by the CFD code. It is called for each boundary node within the CFD calculations until convergence to a steady solution is reached. The numerical approach is used to investigate the effect of flow pattern on permeate fluxes in a locally designed and fabricated direct contact membrane distillation (DCMD) module.

\section{Governing equations in MD}

In MD, liquid enters the feed side at some desired temperature, as depicted in Figure 1(a), while a cooler fluid flows in the permeate side in a counter current (could be in a co-current mode). A liquid coolant is used in DCMD while a gas, commonly air/water vapor, flows in air gap (AGMD), or vacuum membrane distillation (VMD) configurations [28]. In the latter, the pressure in the permeate side is also lowered in order to enhance species transfer.

MD consists of transferring chemical species in vapor state from a feed side to a permeate side through a micro-porous hydrophobic membrane. The rate of species transferred is driven by the temperature difference between the two sides of the membrane with the feed side being the hot stream. Detailed description of the MD process has been widely reported elsewhere [29-33].

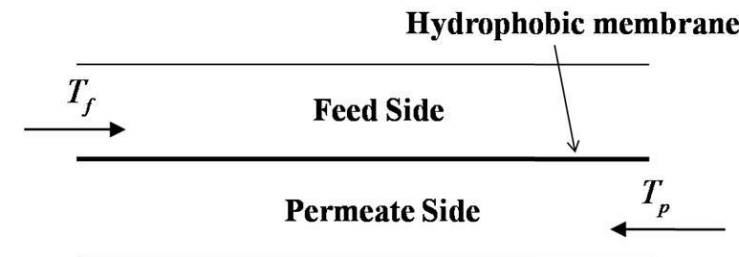

(a) Process scale

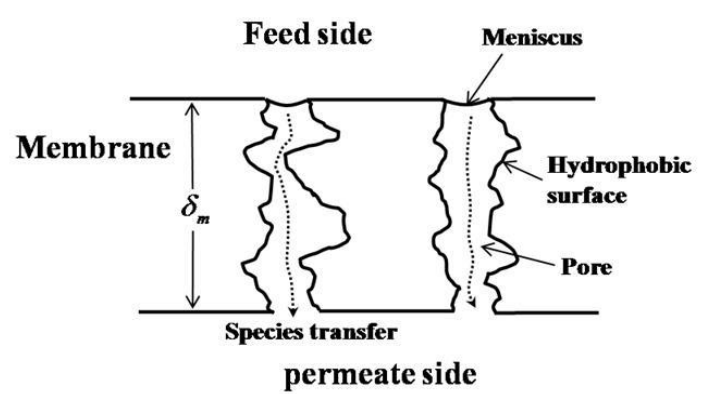

(b) Membrane pore scale

Figure 1. Schematic of direct contact membrane distillation (DCMD): a) process scale, and b) membrane pore scale.

The hydrophobicity of the membrane prevents the liquid feed from entering the pores. However, due to non-equilibrium conditions between the two sides, the most volatile species escape the meniscus formed at pores inlet and flow through the porous membrane in a vapor state to reach the permeate side and condense (see Figure 1(b)). 


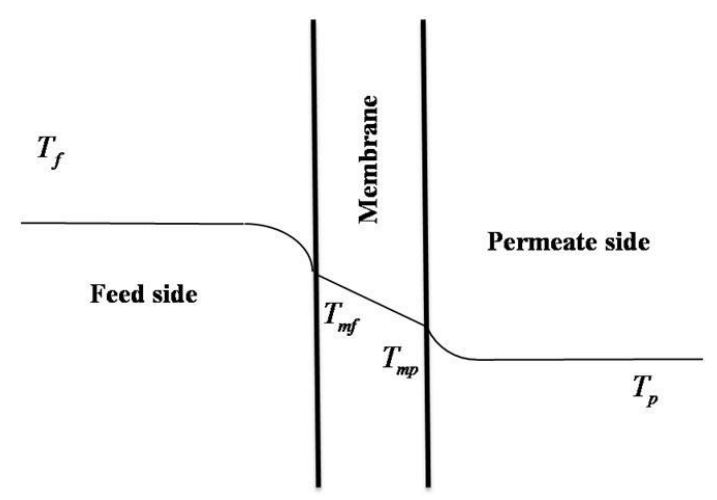

Figure 2. Temperature evolution from feed to permeate in membrane distillation.

Ideally, the surface temperature on the feed side of the membrane equals the feed temperature $T_{f}$ and the surface temperature on the permeate side of the membrane equals the bulk temperature $T_{p}$ of the condensing fluid. However, the process is known to suffer from temperature polarization as depicted in Figure 2 causing a decrease in permeate fluxes [34]. The temperature drops from the bulk value of the feed to a lower surface temperature $T_{m f}$ on the feed side of the membrane, to further decrease to a surface temperature $T_{m p}$ on the permeate side of the membrane and finally to the bulk temperature of the permeate side $T_{p}$ (desired/controlled coolant temperature).

A recent review by Hitsov et al. [35] reports the current models for mass transfer inside the membrane. The rate of species transfer from the feed side to the permeate side is often expressed as:

$$
J=C \Delta P=C\left(P_{m f}-P_{m p}\right)
$$

where $C$ is a mass transfer coefficient that depends on the nature of the species transferred, the membrane properties and the operating conditions, $\Delta P$ represents the difference between the vapor pressure $P_{m f}$ at the meniscus and the vapor pressure $P_{m p}$ at the permeate side of the membrane.

Considering DCMD, species transport across the porous membrane often lies in a mixed Knudsen-ordinary diffusion regime, with the coefficient $C$ given by [36-38]:

$$
C=\left(\frac{3 \xi \delta_{m}}{2 \varepsilon_{m} r} \sqrt{\frac{\pi R T_{a v}}{8 M}}+\frac{\xi \delta_{m}}{\varepsilon_{m}} \frac{P_{a}}{P D_{s}} \frac{R T_{a v}}{M}\right)^{-1}
$$


where $\xi$ is the membrane tortuosity, $\varepsilon_{m}$ is the membrane porosity, $\delta_{m}$ is the membrane thickness, $P$ is the total pressure at the pore entrance, $D_{s}$ is the diffusion coefficient of the liquid species to be transferred, $P_{a}$ is the air pressure inside the pore (close to atmospheric pressure in DCMD [39]) and $T_{a v}$ is the average temperature inside the pore taken as $T_{a v}=\left(T_{m f}+T_{m p}\right) / 2$. The product of the total pressure and the diffusion coefficient is a function of temperature, viz. [37, 39]

$$
P D_{s}=1.895810^{-5} T^{2.072}
$$

The contributions (Knudsen vs. diffusion) to species transport are more or less important depending on the MD process configuration (DCMD, VMD or AGMD).

The vapor pressure is commonly calculated knowing the local temperature via Antoine's equation, viz.

$$
P=\exp \left(a-\frac{b}{T-c}\right)
$$

The coefficients $a, b$ and $c$ depend on the nature of the species. For pure water, these coefficients are $a=23.1964, b=3816.44$ and $c=46.13$ [36]. However, Eq. (4) is valid only for pure components. In the case of seawater, water salinity " $s$ " affects vapor pressure, a correction for calculations on the feed side is consequently brought to Eq.(4), viz,

$$
P_{s w}=\frac{P}{1+0.57357\left(\frac{s}{1000-s}\right)}
$$

\section{Modeling approach}

In the present analysis, turbulent non-isothermal flow conditions prevail in the MD module and the Reynolds averaged equations with the RNG $k-\varepsilon$ turbulent model of ANSYS fluent software [27] are solved. Regarding mass transfer calculations, seawater is considered as a mixture of $\mathrm{H}_{2} \mathrm{O}$ and $\mathrm{NaCl}$ species. Species transport is modeled using a single phase multi-component approach with no bulk reaction. The governing equations for momentum, heat and mass transfer are reported in Appendix A. The dependence of seawater physical properties on temperature is taken into account as reported in the sequel. These properties are introduced in ANSYS fluent via DEFINE_PROPERTY user defined functions (UDF). 
The coupling strategy between the feed side, the pore scale and the permeate side condition is illustrated in Figure 3. A temperature is imposed at the domain inlet and a convective heat transfer condition is assigned to the membrane surface. The temperature at the domain boundary as given by CFD calculations is assumed to be $T_{m f}$ and the membrane thickness is assumed to virtually extend beyond the computational grid. Therefore, the surface temperature $T_{m p}$ on the permeate side of the membrane remains unknown. It will be determined by solving the transport equations at the membrane scale as explained in the sequel.

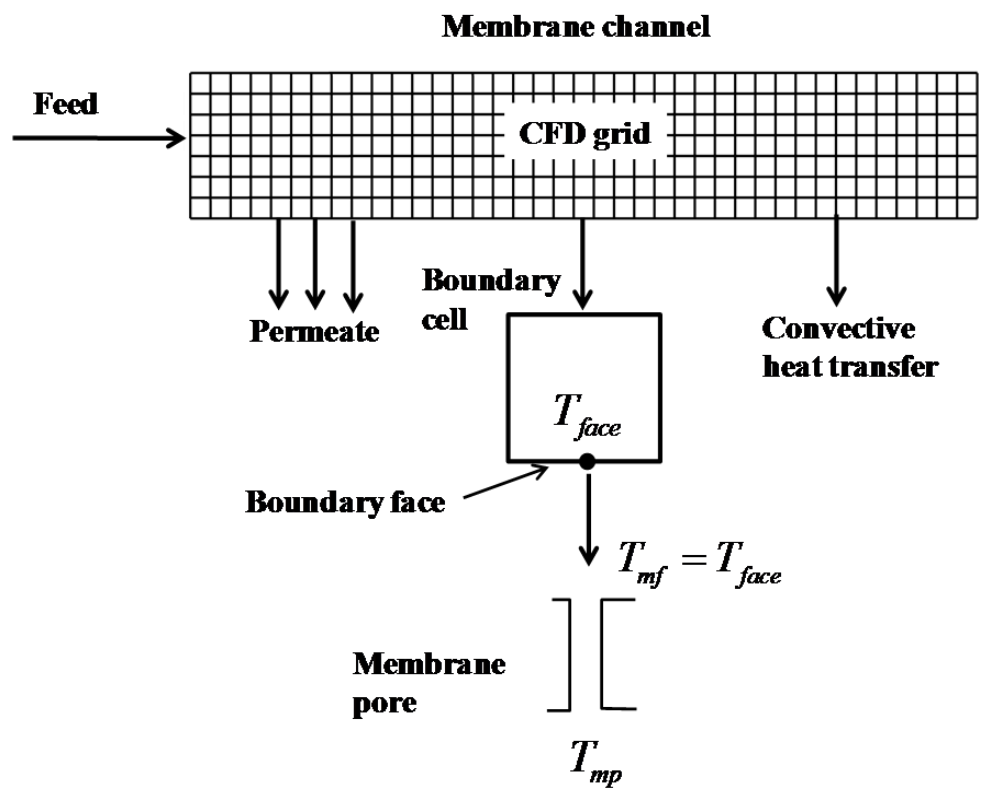

Figure 3. Calling the membrane scale procedure from CFD boundary cells.

The convective boundary condition is commonly expressed as:

$$
Q=h_{e x t}\left(T_{w}-T_{e x t}\right)
$$

where $h_{e x t}$ is the external heat transfer coefficient, $T_{w}$ is the boundary temperature and $T_{\text {ext }}$ is the bulk temperature.

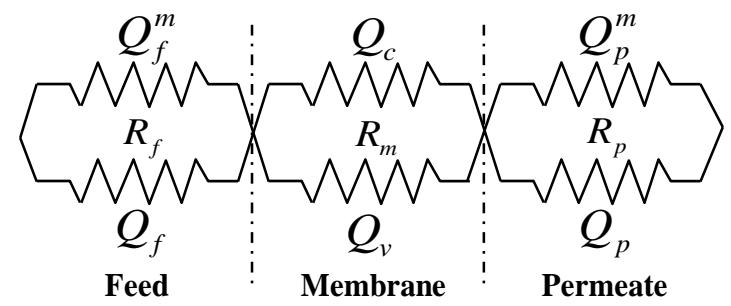

Figure 4. Temperature polarization in membrane distillation: Electrical analog. 
Since the condition is applied at the interface between the feed and the membrane, additional resistance to convective heat transfer has to be taken into account as depicted in the electric analog of Figure 4 [40]. The resulting external heat transfer coefficient can be expressed as:

$$
h_{e x t}=\left(\frac{1}{R_{m}}+\frac{1}{R_{p}}\right)^{-1}
$$

where $R_{m}$ represents the membrane total resistance and $R_{p}$ the permeate side resistance.

The heat transfer through the porous membrane is a contribution of conduction and species transport from the feed to the permeate side of which the equivalent resistance $R_{m}$ can be expressed as [39, 41]:

$$
R_{m}=\frac{1}{\frac{\lambda_{m}}{\delta_{m}}+\frac{J H_{v}}{T_{m f}-T_{m p}}}
$$

where $\delta_{m}$ is the membrane's thickness, $H_{v}$ is the vapor enthalpy, $\lambda_{m}$ is the thermal conductivity of the membrane and $J$ is the rate of species transferred across the membrane.

Vapor enthalpy is a function of vapor temperature and can be expressed in SI units as [42]:

$$
H_{v}=1753.5 T+2024.3 \times 10^{3}
$$

where $T$ is an average temperature between feed and permeate sides.

The resistance to heat transfer in the permeate side is a parallel contribution of convective transfer and condensation and can be readily expressed as:

$$
R_{p}=h+\frac{J H_{L}}{T_{m p}-T_{p}}
$$

where $h$ represents the convective heat transfer coefficient and $H_{L}$ is the enthalpy of the permeate solution evaluated at the mean temperature of the permeate bulk temperature and the membrane surface temperature on the permeate side [40].

The enthalpy of the permeate solution as a function of temperature in SI units is given by:

$$
H_{L}=4186.3 T-1143.4 \times 10^{3}
$$

The convective heat transfer coefficient defined by the flow conditions on the permeate side is usually calculated from the Nusselt number $\mathrm{Nu}$. The latter is given by: 


$$
\mathrm{Nu}=\frac{h l}{\lambda_{f}}
$$

where $l$ represents a characteristic length and $\lambda_{f}$ the thermal conductivity of the fluid.

Under turbulent flow conditions, the Nusselt number can be estimated using the following correlation [43]:

$$
\mathrm{Nu}=0.036 \operatorname{Re}^{0.8} \operatorname{Pr}^{1 / 3}\left(\frac{d_{h}}{l}\right)^{0.055}
$$

where Re represents the Reynolds number, $\operatorname{Pr}$ is the Prandtl number, $d_{h}$ the hydraulic diameter and $l$ a characteristic length.

On the permeate side, seawater flows at $20^{\circ} \mathrm{C}$ and at a rate of $1.5 \mathrm{LPM}$. These conditions are used to estimate the convective heat transfer coefficient at the permeate side. The value of $h$ retained is $20,000 \mathrm{~W} \cdot \mathrm{m}^{-2} \cdot \mathrm{K}^{-1}$.

Given a computational domain, the temperature, the fluid velocity and the species mass fraction are set at the inlet. An adiabatic condition is assigned to all wall boundaries except the membrane surface where the convective heat transfer condition is imposed with the modified heat transfer coefficient given by Eq. (7) as well as a flux condition for species consumption set by Eq. (1). The modification of the heat transfer coefficient is carried out within the ADJUST_PROFILE UDF while the consumption rate is considered as a surface reaction and consequently implemented in DEFINE_SR_RATE UDF provided by ANSYSfluent [27]. Both routines are called for each boundary face (see Figure 3). However, the modification of the heat transfer coefficient using Eq. (7) in the CFD model is subject to the knowledge of temperatures $T_{m f}$ and $T_{m p}$ and salinity, and these can only be determined if the transport problems at both the membrane and channel scales are solved simultaneously.

In order to update the permeate side temperature of the membrane at runtime, a heat balance across the membrane is considered. At steady state, the flux transferred from the feed to the membrane equals the flux that passes through the membrane as well as the flux transferred to the permeate side (see figure 4), viz.

$$
Q_{f}+Q_{f}^{m}=Q_{c}+Q_{v}=Q_{p}+Q_{p}^{m}
$$

where $Q_{f}$ is the convective heat transferred from the feed to the membrane, $Q_{f}^{m}$ is the heat due to mass transfer from the feed to the membrane, $Q_{c}$ is the heat transferred by conduction across the membrane, $Q_{v}$ is the heat due to vapor transport across the membrane, $Q_{p}$ is the 
heat transferred from the membrane surface to the permeate side, and $Q_{p}^{m}$ is the heat due to mass transfer from the membrane to the permeate side.

Using the temperature distribution across the membrane derived by Phattaranawik et al. $[40,44]$, the total flux through the membrane can be expressed as:

$$
Q_{m}=Q_{c}+Q_{v}=\frac{c_{p v} J T_{m f} z-c_{p v} J T_{m p}}{z-1}+J\left(H_{v}-c_{p v} T_{r e f}\right)
$$

with $z$ given by:

$$
z=\exp \left(\frac{J c_{p v} \delta_{m}}{\lambda_{m}}\right)
$$

Rearranging leads to:

$$
T_{m p}-T_{m f} z+(z-1) \frac{\left[Q_{m}-J\left(H_{v}-c_{p v} T_{r e f}\right)\right]}{c_{p v} J}=0
$$

Since at steady state $Q_{m}=Q_{p}+Q_{p}^{m}$, one can write:

$$
f\left(T_{m p}\right)=T_{m p}-T_{m f} z+(z-1) \frac{\left[h\left(T_{m p}-T_{p}\right)+J H_{L}-J\left(H_{v}-c_{p v} T_{r e f}\right)\right]}{c_{p v} J}=0
$$

The species flux $J$ as given by Eq. (1) is strongly dependent on temperatures $T_{m f}$ and $T_{m p}$ and local seawater salinity via the vapor pressures calculated using Eq.(5), while $H_{v}$ and $H_{L}$ are estimated with equations (9) and ( 11) respectively. Equation (18) exhibits a strong nonlinearity with respect to the unknown temperature $T_{m p}$ and is solved via an in-house iterative procedure called from the CFD solver. At each boundary face, Eq. (18) is solved using Quasi-Newton method between temperatures $T_{\text {face }}=T_{m f}$ and $T_{p}$. This choice of the temperature interval to start the non-linear solver iterations ensures robustness of the procedure which is crucial for a successful simulation. Indeed, the non-linear solver is called from the CFD code for each boundary face until the overall convergence of the flow, heat and mass transfer solution is reached. Numerical experiments have shown that the solution for each boundary node is reached within few iterations. The pressure-based coupled solver of ANSYS-fluent, of which general algorithm is represented in Figure 5 is selected to calculate the momentum, heat and mass transfer solution. During the iterative CFD calculations, the DEFINE_ADJUST UDF is called for each boundary face to calculate the surface temperature on the permeate side of the membrane. Using the local value of salinity, it solves Eq. (18) with the integrated non-linear solver, and update the heat transfer coefficient of Eq.(7). The 
resulting permeate flux is used in DEFINE_SR_RATE UDF to set the boundary condition for species transport. The solution returns with updated values of species mass fraction, thus salinity. The latter, with the new temperature value is used by the DEFINE_PROPERTY UDFs to update the fluid physical properties. The whole procedure is repeated until global model convergence is reached. The simple solver was selected for all calculations with an accuracy criterion of $10^{-4}$ for all momentum variables and mass conservation, while a criterion of $10^{-6}$ was used for temperature and species mass fraction.

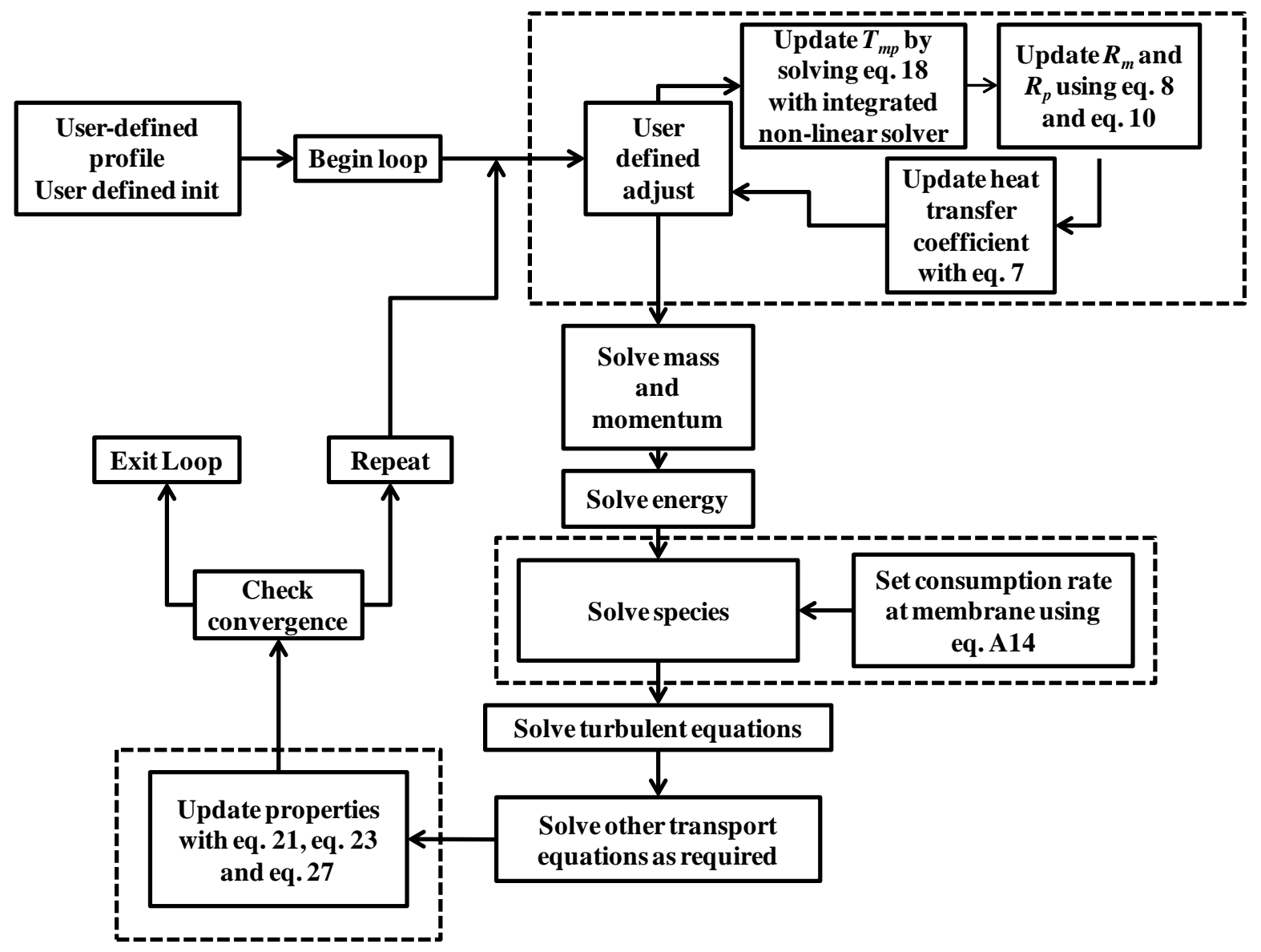

Figure 5. Solution procedure of the coupled CFD-pore scale approach [27]. (Embedded in-house procedures appear inside dashed squares).

\section{Experimental procedure}

A schematic diagram of the used custom-made automated DCMD experimental setup designed and fabricated in our workshop is presented in Figure 6 [31]. The active membrane surface area (A) inserted in a flat sheet module fabricated using poly methyl methacrylate material is $0.005 \mathrm{~m}^{2}$ (flow channel dimensions of $0.1 \mathrm{~m} \times 0.05 \mathrm{~m} \times 0.002 \mathrm{~m}$ ). Two different 
membranes were used for DCMD testing. The first membrane, denoted M1 in the sequel, is a composite membrane with a poly tetra fluoroethylene (PTFE) active layer and a nonwoven polyester support. The second membrane, denoted M2, is composite membrane with a PTFE active layer and a scrim backing polypropylene support.

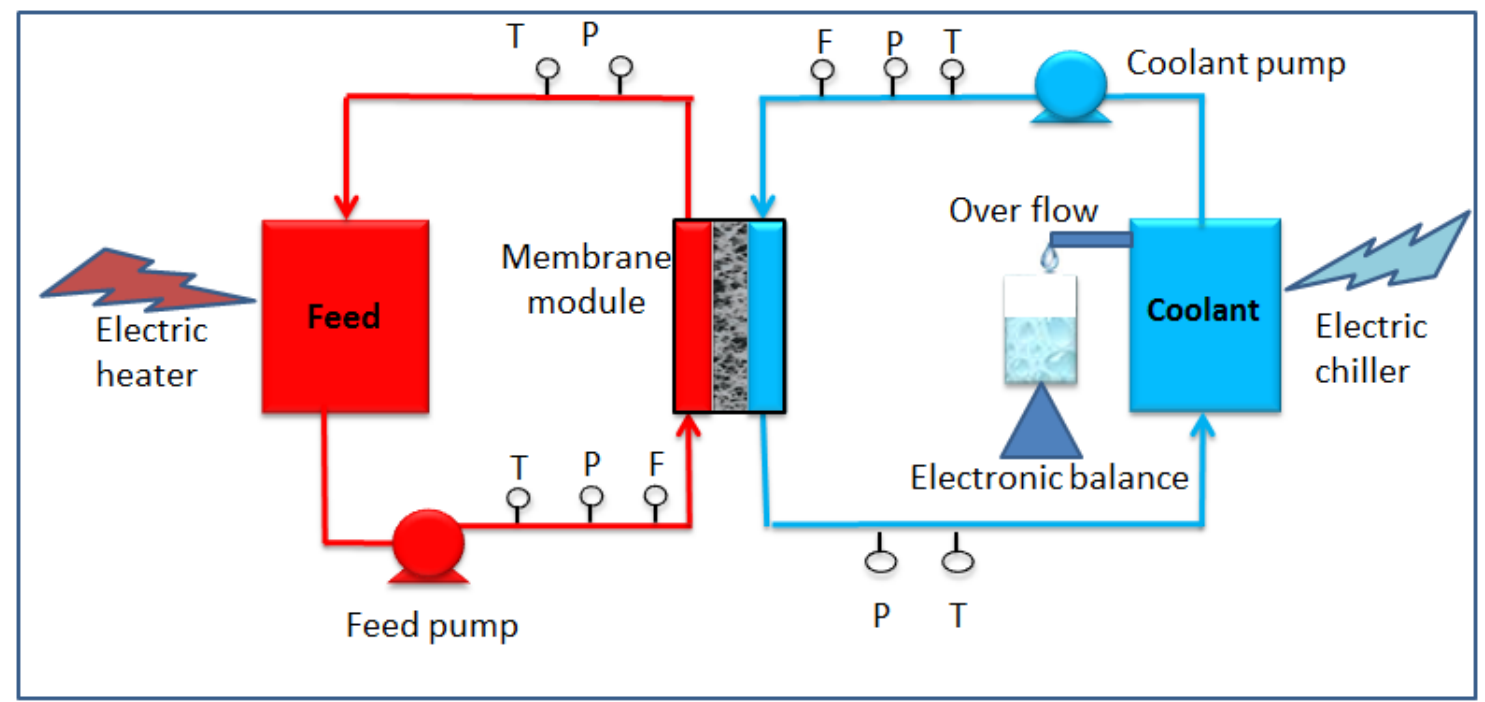

Figure 6. Schematic of the experimental setup.

The hot feed seawater with a salinity of $4.2 \mathrm{wt} \%$ and the coolant (Milli-Q water) were circulated in both sides of the MD membrane in a counter current manner. Temperatures were controlled using thermo-regulators inserted in the feed and coolant tanks. Conductivities of feed and permeate collected from the overflow of the coolant tank were monitored using Oakton Eutech Instruments, Malaysia, conductivity meters. The permeate container was placed on a very accurate balance connected to a lab view software. The flux $J_{v}$ was determined by: $J_{v}=m_{w} / A t$ ( $m_{w}$ is the mass of collected permeate at a particular time interval t). All experiments were run at constant feed and coolant flow rates of $1.5 \mathrm{~L} / \mathrm{min}$.

\section{Membrane and fluid properties}

Details of membranes characteristics determined experimentally and their performance for different feed water quality, e.g., seawater desalination, were reported elsewhere, e.g. [31, 45]. Some of the PTFE membranes characteristics are presented in Table 1, while unavailable properties are estimated using appropriate correlations. 
Table 1. PTFE membranes characteristics.

\begin{tabular}{lll}
\hline Property & M1 & M2 \\
\hline Thickness, $\delta_{m}\left(\mu_{\mathrm{m}}\right)$ & $170 \pm 4$ & $100 \pm 2$ \\
Porosity, $\varepsilon_{m}$ & 0.73 & 0.68 \\
Mean flow pore size, $r\left(\mu_{\mathrm{m}}\right)$ & 0.26 & 0.5 \\
\hline
\end{tabular}

\section{Membrane tortuosity}

The tortuosity $\xi$ of the membrane is determined from the porosity using the following relationship [46]:

$$
\xi=1-0.8 \ln \left(\varepsilon_{m}\right)
$$

which gives a tortuosity of 1.25 for membrane M1 and a tortuosity of 1.31 for membrane M2.

\section{Membrane thermal conductivity}

The isostress model is used to estimate the thermal conductivity of the membrane. The model has been shown as the most appropriate for MD membranes [44] and gives the thermal conductivity as a function of the thermal conductivities of the porous material and the gas occupying the pores, viz.

$$
\lambda_{m}=\left(\frac{\varepsilon_{m}}{\lambda_{g}}+\frac{1-\varepsilon_{m}}{\lambda_{s}}\right)^{-1}
$$

where $\lambda_{s}$ is the thermal conductivity of the solid phase and $\lambda_{g}$ is the thermal conductivity of the fluid phase.

The thermal conductivity of the composite membrane-air is calculated using the thermal conductivity of PTFE $(0.25 \mathrm{~W} / \mathrm{m} / \mathrm{K}$ [47]), the thermal conductivity of air $(0.0234 \mathrm{~W} / \mathrm{m} / \mathrm{K})$ and membrane porosities of 0.73 and 0.68 , respectively (as reported in Table 1). The resulting thermal conductivities of membrane $\mathrm{M} 1$ and membrane $\mathrm{M} 2$ are $0.031 \mathrm{~W} / \mathrm{m} / \mathrm{K}$ and $0.033 \mathrm{~W} / \mathrm{m} / \mathrm{K}$, respectively.

\section{Seawater specific heat}

Seawater specific heat at constant pressure is estimated using the following equation [28, 48]: 


$$
c_{p}=10^{-3}\left(A_{c_{p}}+B_{c_{p}} T+C_{c_{p}} T^{2}+D_{c_{p}} T^{3}\right)
$$

where,

$$
\begin{aligned}
& A_{c_{p}}=4206.8-6.6197 S+1.228810^{-2} s^{2} \\
& B_{c_{p}}=-1.1262+5.417810^{-2} s-2.271910^{-4} s^{2} \\
& C_{c_{p}}=1.202610^{-2}-5.356610^{-4} s+1.890610^{-6} s^{2} \\
& D_{c_{p}}=6.877710^{-7}+1.51710^{-6} s-4.426810^{-9} s^{2}
\end{aligned}
$$

Eq. (35) is valid for temperatures ranging from $10^{\circ} \mathrm{C}$ to $180^{\circ} \mathrm{C}$ and salinity ranging from 20 g. $\mathrm{Kg}^{-1}$ and $160 \mathrm{~g} . \mathrm{Kg}^{-1}$.

\section{Seawater viscosity}

The viscosity of seawater as a function of temperature can be approximated within $\pm 1 \%$ for temperatures between $20^{\circ} \mathrm{C}$ and $180^{\circ} \mathrm{C}$ and salinity ranging from 0 g. $\mathrm{Kg}^{-1}$ to $130 \mathrm{~g} . \mathrm{Kg}^{-1}$ by the following equation in SI units $[28,48]$ :

$$
\mu_{s w}=0.001 \mu_{w} \mu_{r}
$$

where $\mu_{M}$ and $\mu_{R}$ are given by:

$$
\ln \left(\mu_{w}\right)=-3.79418+\frac{604.129}{139.18+T}
$$

and

$$
\mu_{r}=1+A_{\mu} s+B_{\mu} s^{2}
$$

with,

$$
\begin{aligned}
& A_{\mu}=1.47410^{-3}+1.510^{-6} T-3.92710^{-8} T^{2} \\
& B_{\mu}=1.073410^{-5}-8.510^{-8} T+2.2310^{-10} T^{2}
\end{aligned}
$$

\section{Seawater thermal conductivity}

The thermal conductivity $\lambda_{s w}$ of seawater for temperatures between $0 .{ }^{\circ} \mathrm{C}$ and $180^{\circ} \mathrm{C}$ and salinity up to $160 \mathrm{~g} \cdot \mathrm{Kg}^{-1}$ can be estimated in $\mathrm{mW} \cdot \mathrm{m}^{-1} \cdot \mathrm{K}^{-1}$ within an accuracy of $\pm 3 \%$ using $[48,49]$ :

$$
\log _{10}\left(\lambda_{s w}\right)=\log _{10}\left(240+2.10^{-4} s\right)+0.434\left(2.3-\frac{343.5+0.037 s}{T+273.15}\right)\left(1-\frac{T+273.15}{647+0.03 s}\right)^{0.333}
$$




\section{$\mathrm{NaCl}$ diffusion coefficient}

The diffusion coefficient $D^{(\text {salt })}$ of $\mathrm{NaCl}$ is determined from its ions $\mathrm{Na}^{+}$and $\mathrm{Cl}^{-}$ diffusivities at infinite dilution using [50]:

$$
D^{(\text {Salt })}=\frac{\left(z_{+}+\left|z_{-}\right|\right) D^{\left(\mathrm{Na}^{+}\right)} D^{\left(\mathrm{Cl}^{-}\right)}}{z_{+} D^{\left(\mathrm{Na}^{+}\right)}+\left|z_{-}\right| D^{(\mathrm{Cl})}}
$$

where $z_{+}$and $z_{-}$represent the charges of $\mathrm{Na}^{+}$and $\mathrm{Cl}^{-}$ions respectively, namely, +1 and -1 . Using a value of $1.33410^{-9} \mathrm{~m}^{2} . \mathrm{s}^{-1}$ for $\mathrm{Na}^{+}$and $2.03210^{-9} \mathrm{~m}^{2} \cdot \mathrm{s}^{-1}$ for $\mathrm{Cl}^{-}$[50], the diffusion coefficient of "Salt"species is set to $1.6210^{-9} \mathrm{~m}^{2} \cdot \mathrm{s}^{-1}$.

\section{Numerical simulation set-up}

\subsection{Boundary conditions:}

The feed side of the MD module is of parallelepipedic shape with a height of $2 \mathrm{~mm}$, a width of $50 \mathrm{~mm}$ and a length of $100 \mathrm{~mm}$. The feed inlet and outlet are located at two opposite corners of the parallelepiped, as depicted in Figure 7. The boundary conditions on this model can be summarized as:

- A flat velocity profile at the inlet.

- Constant temperature at the inlet.

- No slip boundary condition at all domain walls.

- Adiabatic condition at all domain walls except the membrane's surface. The MD module is made of a shell much thicker than the feed channel which enables to assume an ideal insulation.

- A convective (mixed type) condition at the membrane's surface with a heat transfer coefficient corrected using membrane pore scale calculations.

- An outflow condition at the outlet.

- Constant species mass fraction at the inlet.

- A flux condition for water species at the membrane's surface, given by the rate of transferred species from the feed to the permeate side (see governing equations in Appendix A). 


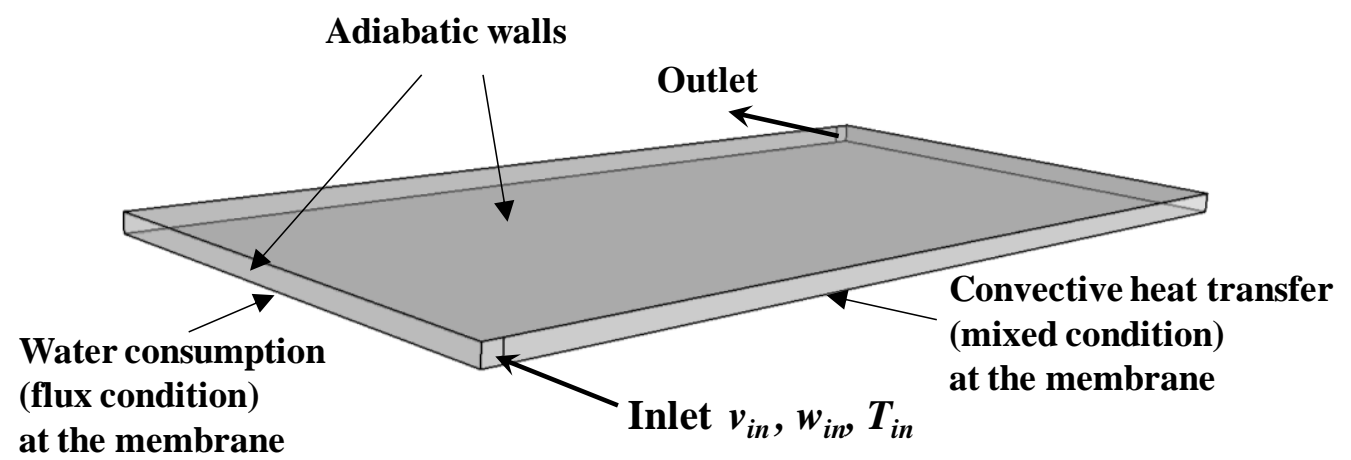

Figure 7. Module scale boundary conditions.

\subsection{Mesh generation}

The first step in the set up of the numerical model consists of meshing the MD module geometry. Mesh generation represents a crucial part of simulations' success and particular consideration should be taken in case of turbulent flow. The main task resides in the resolution of the boundary layer. The latter represents the distant from the wall from which the viscous effects become negligible. It is known that the boundary layer consists of different regions, namely, the viscous sublayer where the flow is assumed laminar and the log layer where turbulent flow dominate, with a transition region between the two, called the buffer layer [51]. From the numerical standpoint, different models are used to describe the viscous and the log layers while models generally fail to represent properly the flow in the transition region. Consequently, mesh generation needs to make sure that no computational nodes lie within this region [27]. The distance above the wall is generally described using a dimensionless height $y^{+}=\rho_{w} u_{\tau} y / \mu_{w}$, where $\rho_{w}$ is the fluid density near the wall, $\mu_{w}$ is the fluid viscosity near the wall, $y$ is the height above the wall, and $u_{\tau}$ is the friction velocity. The latter is expressed as $u_{\tau}=\sqrt{\tau_{w} / \rho_{w}}$ with $\tau_{w}$ is the wall shear stress. The dimensionless distance $y^{+}$can be used to help set the first mesh layer above the wall. It is established that $30<y^{+}<300$ corresponds to the $\log$ law region and $y^{+}<5$ to the viscous sublayer, although some authors reported intervals up to $y+<10[52,53]$. Consequently, values 5 to $10<y^{+}<30$ correspond to the buffer region. Unfortunately, in the case of the confined flow undertaken in this article, preliminary calculations reveal the presence of recirculation loops and different flow zones with large velocity variations. At the center of recirculation zones, wall shear stress tends to negligible values which lead to small $y^{+}$coordinates. On the other hand, for 
the highest velocity value, preliminary calculation for the condition $y^{+}>30$ suggests a first cell height having the same order of magnitude as the channel height which leads to an unrealistically coarse mesh unable to resolve dead zones and centers of recirculation loops. Therefore, the dimensionless coordinate exhibits significant variations throughout the MD module and each position on the membrane surface requires a different value. Moreover, the simple parallelepipedic shape of the module suggests the construction of a structured mesh that allows a precise control of the extrusion of a base planar mesh as well as elements stretching. It is therefore more convenient to refine a structured mesh up to a desired accuracy and verify by post-processing that $y^{+}$remains below a given value throughout the domain.

In order to make sure that the numerical solution is independent of mesh size, it is suggested to refine the mesh up to the level where no more changes in minimum and maximum values as well as area weighted average values of permeate flux are observed. In the current investigation, the permeate flux is the most stringent reference parameter for grid dependency test. The choice is encouraged by the fact that the variable encompasses implicitly of three transfer modes, namely momentum heat and mass as well as the value of the permeate side temperature of the membrane surface which is computed via an in-house nonlinear solver. Consequently, the permeate flux value depends on the resolution of the different boundary layers and its stabilization will help set the mesh resolution.

Mesh generation in practical applications is based on a tradeoff between accuracy and computational time. The grid dependency test is therefore carried out by progressive refinement of an initial mesh, using GAMBIT [27], and comparison with experimental data as well as results obtained between successive meshes. In this paper, the experimental case with the highest feed inlet temperature and highest observed permeate flux is considered for the grid dependency test (Membrane M2, inlet temperature $80^{\circ} \mathrm{C}$ ). Indeed, highest flux cause steep temperature and species concentration gradients towards the membrane surface and proper boundary resolution becomes more important. The parallelepiped is geometrically subdivided in three main zones as depicted in the top view of Figure 8. The left and right zones are connected to the inlet and outlet. The test starts with a planar mesh of 20,000 nodes in the central region (100x200) and 15 nodes at the lateral regions. The first mesh is extruded in 15 layers in the perpendicular direction. The extrusion is carried out with a successive ratio of 1.2 in double-sided fashion toward both planar walls of the module. The central zone is kept at the same resolution while lateral and transverse layers are increased by 3 nodes for 
each test. Table 2 gathers the minimum, the maximum and the area weighted average of the permeate flux that were post-processed for each mesh. It can be seen that for 27 transversal layers, the value of the maximum flux and the average flux stabilize. However, the minimum value fluctuates, which suggests that center of recirculation loops or dead zones are not well resolved yet. The resolution of the planar mesh is then increased by adding nodes to the central zone. The mesh refinement is stopped at a total number of nodes of $1,171,800$, for which changes in minimum, maximum and area weighted average permeate fluxes remain below $1 \%$ compared to the penultimate mesh.

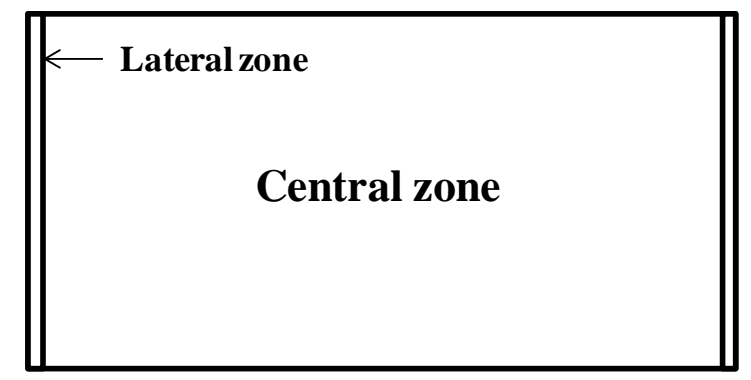

Figure 8. Domain decomposition of the DCMD module (Top view).

Table 2. Grid dependency test results.

\begin{tabular}{cccccccccc}
\hline Mesh ID & 1 & 2 & 3 & 4 & 5 & 6 & 7 & 8 & 9 \\
\hline $\begin{array}{c}\text { Each } \\
\text { lateral } \\
\text { zone }\end{array}$ & 15 & 18 & 21 & 24 & 27 & 30 & 30 & 30 & 30 \\
\hline $\begin{array}{c}\text { Central } \\
\text { zone }\end{array}$ & $\begin{array}{c}100 \times 200 \\
(20000)\end{array}$ & $\begin{array}{c}100 \times 200 \\
(20000)\end{array}$ & $\begin{array}{c}100 \times 200 \\
(20000)\end{array}$ & $\begin{array}{c}100 \times 200 \\
(20000)\end{array}$ & $\begin{array}{c}100 \times 200 \\
(20000)\end{array}$ & $\begin{array}{c}100 \times 200 \\
(20000)\end{array}$ & $\begin{array}{c}108 \times 216 \\
(23328)\end{array}$ & $\begin{array}{c}116 \times 232 \\
(26912)\end{array}$ & $\begin{array}{c}126 \times 250 \\
(31500)\end{array}$ \\
\hline $\begin{array}{c}\text { Extrusion } \\
\text { layers }\end{array}$ & 15 & 18 & 21 & 24 & 27 & 30 & 30 & 30 & 30 \\
\hline nodes & 345000 & 424800 & 508200 & 595200 & 685800 & 780000 & 894240 & 1016160 & 1171800 \\
\hline $\begin{array}{c}\text { Min } \\
\text { permeate } \\
\text { flux }\end{array}$ & 22.8 & 42.9 & 36.2 & 17.6 & 30.9 & 25.1 & 55.7 & 47.9 & 48.1 \\
\hline $\begin{array}{c}\text { Max } \\
\text { permeate } \\
\text { flux }\end{array}$ & 91.3 & 126.5 & 132.3 & 133.7 & 134.7 & 135.7 & 135.6 & 135.7 & 135.7 \\
\hline $\begin{array}{c}\text { Weighted } \\
\text { average } \\
\text { permeate } \\
\text { flux }\end{array}$ & 62.8 & 84.64 & 85.4 & 86.9 & 88.3 & 88.7 & 90.1 & 91.2 & 91.9 \\
\hline
\end{tabular}

The final mesh presented in Fig. 9 shows the refinement at the module edges and the progressive coarsening towards the center of the module (Fig. 9(a)) while Fig. 9(b) shows a zoom over one of the module corners with the progressive refinement towards the module upper wall and the membrane surface. 


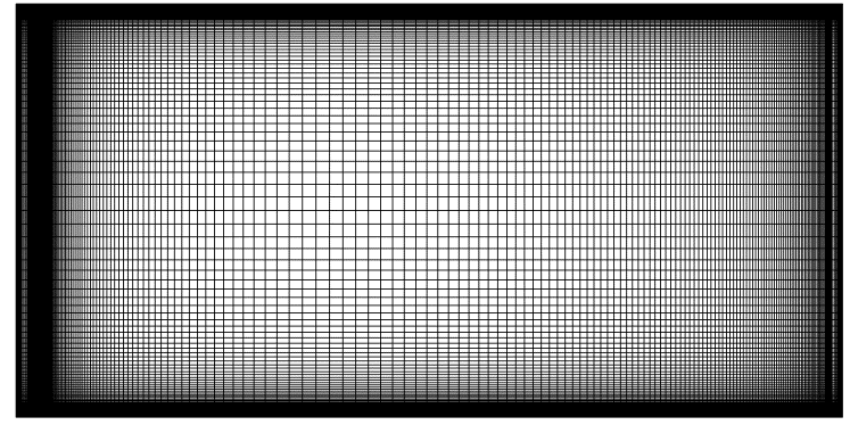

(a)

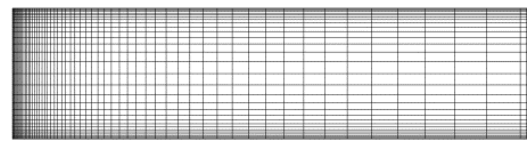

(b)

Figure 9. Mesh of the DCMD module, (a) Top view, (b) Zoom on side view.

Post-processing of the reference case gives the distribution of $y^{+}$dimensionless coordinate distribution over the planar mesh of the membrane (see Fig. 10). The coordinate value is slightly greater than 5 at the vicinity of the fluid inlet and decreases rapidly to reach values as low as 0.1 in some regions of the module. Additional layers could have been used for increased boundary resolution. However, consistency of the numerical results lies primarily in their accordance to experimental results and tradeoff between computational burden and practicality is believed to be reached in this case.

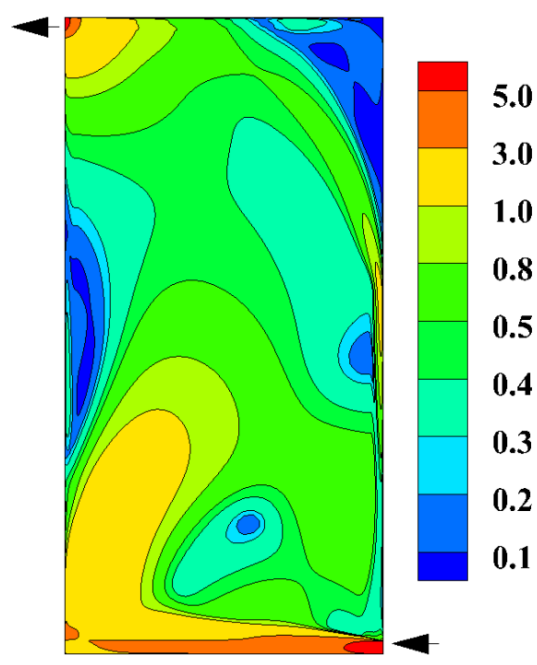

Figure 10. $y^{+}$distribution for the mesh reference case. 


\section{Results and discussion}

\subsection{Comparison of average fluxes}

Experimental results are represented by single average values of the permeate flux for each inlet temperature and should therefore be compared to average simulated permeate fluxes. The fine mesh used for the CFD simulations represents the membrane surface with 39,000 nodes. These boundary nodes are used for the estimation of the average permeate flux. The average flux is weighted by the area of the surface elements of the mesh.

The average values of the experimental and simulated permeate fluxes, at different temperatures, for both $\mathrm{M} 1$ and $\mathrm{M} 2$ membranes are represented in Figure 11. For all temperatures, without any fitting parameter, relative error between experimental values and simulation, as reported in Table 3, remains under 6\%. Therefore, the coupled approach is able to reproduce very closely the average experimental fluxes. Moreover, the representation of the permeate side by an appropriate boundary condition remains valid for this size of prototype module.

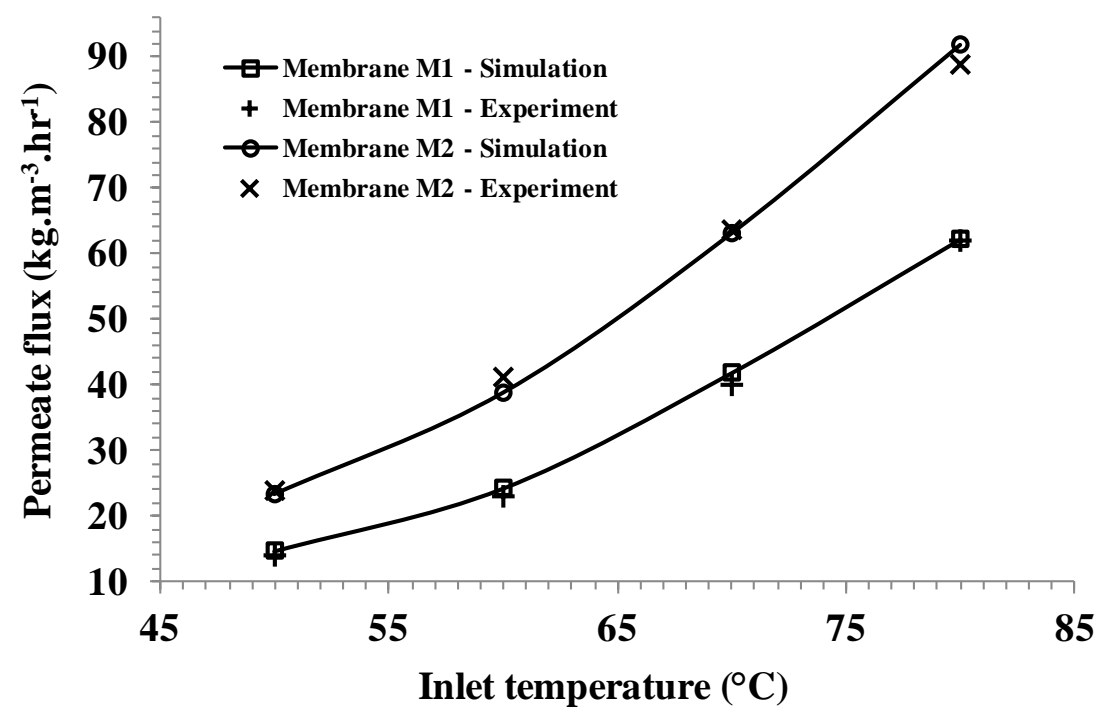

Figure 11. Average permeate flux evolution as a function of inlet temperature for both PTFE membranes.

Table 3. Relative error between experimental and simulation permeate fluxes at different feed inlet temperatures for membrane M1 and membrane M2 (coolant inlet temperature = $\left.20{ }^{\circ} \mathrm{C}\right)$. 


\begin{tabular}{|c|c|c|}
\hline \multirow{2}{*}{ Feed inlet temperature $\left({ }^{\circ} \mathrm{C}\right)$} & \multicolumn{2}{|c|}{ Relative error $(\%)$} \\
\hline & Membrane M1 & Membrane M2 \\
\hline 50 & 5.2 & 2.3 \\
\hline 60 & 5.6 & 5.7 \\
\hline 70 & 4.7 & 0.8 \\
\hline 80 & 0.4 & 3.4 \\
\hline
\end{tabular}

\subsection{Analysis of flow pattern}

The simulation corresponding to an inlet temperature of $80^{\circ} \mathrm{C}$ using membrane $\mathrm{M} 2$ is taken as a base case to analyze the flow and the temperature distributions. The inlet flow rate of 1.5 LPM on the feed side, which corresponds to a Reynolds number of 12,500 at the inlet, generates a turbulent flow and the RNG $k, \varepsilon$ model of ANSYS-fluent is used (see Appendix A). Streamtraces evolution in the MD channel is depicted in Figure 12. The flow has characteristics of a typical impinging jet flow confined between parallel plates. There is abundant literature on the analysis of heat transfer in impinging flows for its industrial importance. Applications span from design of electronic devices, to industrial heat exchangers. The RNG $k, \varepsilon$ model has been shown to perform well in this type of flow [54]. However, investigations often focus on cooling performance. In the present article, heat loss from the feed side is undesired and additional confinement is caused by the module lateral sidewalls, which redirects the complete flow to a single side of the jet. The size of the vortex structure depends strongly on the confinement effect and the inlet velocity. The relatively large distance from the inlet to the facing wall as well as the relatively high velocity at the channel inlet followed by a sudden change in available cross section leads to large recirculation loop "L" near the entrance. The module of the fluid velocity, as represented by the streamtraces colors, decreases slightly after the inlet zone " $\mathrm{C} 1$ " due to the increase of flow cross section. However, due to the significant inlet stream, high velocities still prevail up to corner $\mathrm{C} 2$, then the fluid changes direction along the module diagonal towards corner $\mathrm{C} 4$ where a dead zone is observed. From the center of the channel, the streamlines concentrate toward exit C3 with increasing velocities due to the decrease in flow cross section. The large vortex structure leads to flow detachment along the lateral edge $\mathrm{E}$ due to an adverse pressure gradient caused by the no-slip boundary condition, with the occurrence of a third recirculation loop where low velocities prevail. 


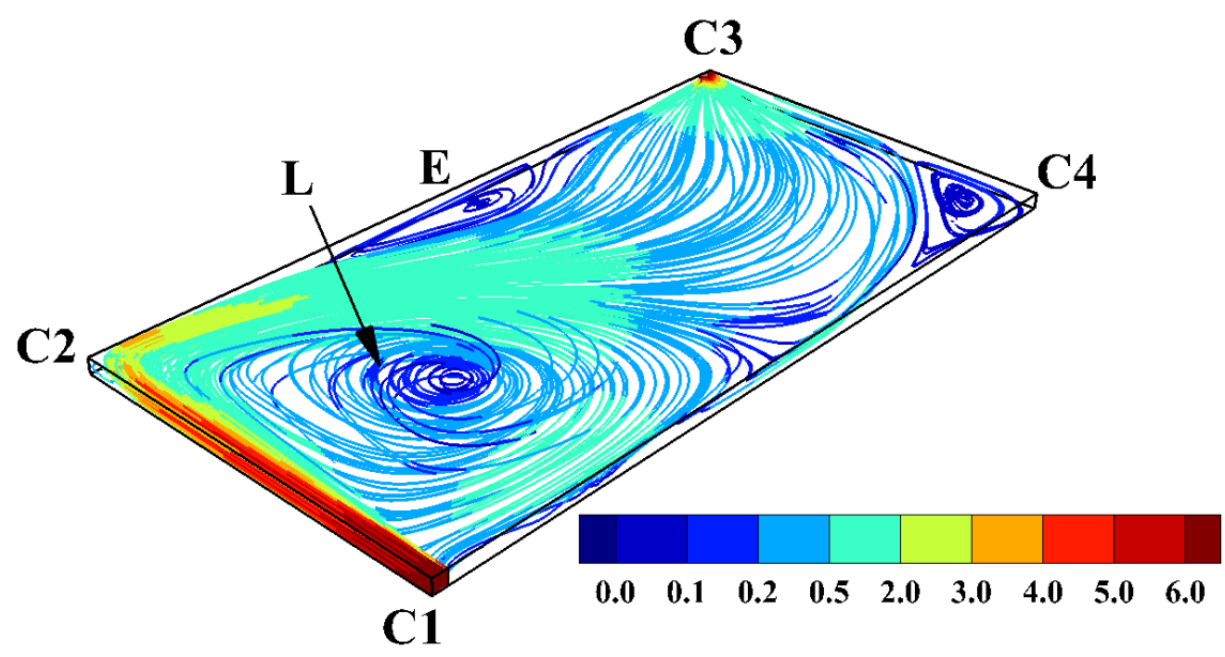

Figure 12. Streamlines in the DCMD module (color by velocity module in $\mathrm{m}^{-\mathrm{s}^{-1}}$ ).

The distribution of the velocity magnitude at $1 \mathrm{~mm}$ above the membrane surface is shown in Figure 13. The values remain significant along the edge from " $\mathrm{C} 1$ " to "C2" and toward the center of the channel. However, an important drop is observed in corner "C4" and edge "E" as well as the recirculation loop close to the inlet. Note that at the center of the module where the velocity is about $0.45 \mathrm{~m} . \mathrm{s}^{-1}$ and using the hydraulic diameter of the rectangular channel (see channel dimensions in experimental section), the Reynolds number remains above 3,400. Therefore, turbulent conditions prevail in the entire module.

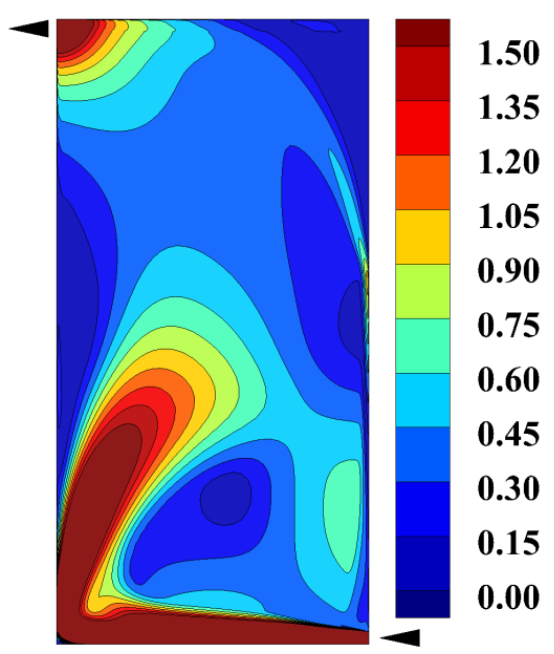

Figure 13 . Velocity module distribution in $\mathrm{m}^{-\mathrm{s}^{-1}} 1 \mathrm{~mm}$ above the membrane surface. 


\subsection{Temperature distribution}

The distribution of the fluid temperature at different heights above the membrane surface is represented in the top view of the DCMD module in Figure 14. The location of the inlet is visible at the right bottom corner of the view and the outlet at the opposite corner. At a height of $2 \mathrm{~mm}$ above the membrane surface (top wall of the channel), in Figure 14(a), the temperature remains close to the feed temperature above the regions where the highest velocities were observed (see Figure 13). However, a $15^{\circ} \mathrm{C}$ drop occurs at the right top corner (C4) where a dead zone exists. The temperature change at the three recirculation zones becomes more important as shown in the horizontal slice at $1 \mathrm{~mm}$ above the membrane surface (Figure 14(b)). The temperature drop is more important near the top right corner due to the creeping flow regime in that region. As the slice gets closer to the membrane surface, namely at a height of $0.5 \mathrm{~mm}$ and $0.1 \mathrm{~mm}$ (see Figure 14(c) and Figure 14(d), respectively), temperature changes drastically. It ranges from $50^{\circ} \mathrm{C}$ at the left edge and top right corner to $80^{\circ} \mathrm{C}$ at the channel inlet. The temperature distribution of Figure 14(d) follows the flow behavior, as one can notice the highest temperature isovalues tends to propagate along the main streamlines towards the exit. Moreover, the lowest temperatures are observed at corner "C4", edge "E" and recirculation loop "L" of Figure 12 which confirms that dead zones and recirculation in DCMD modules can severely limit heat transfer. This is explained by the fact that high tangential velocities minimize the height of the temperature boundary layer thus providing more heat to the separation process. In zones where near stagnation is observed such as center of recirculation loops and corners, the heat transfer process becomes limited by the lack of forced convection and the process separation performance in these zones relies mostly on the fluid thermal conductivity.

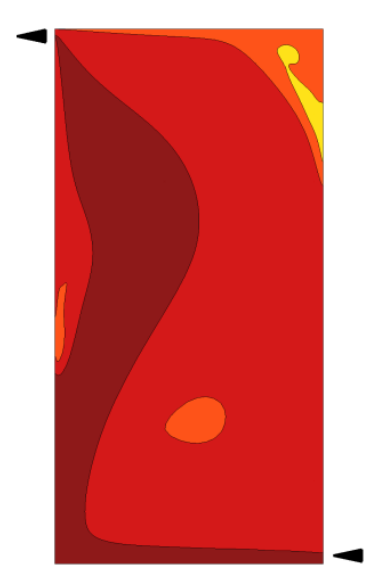

(a)

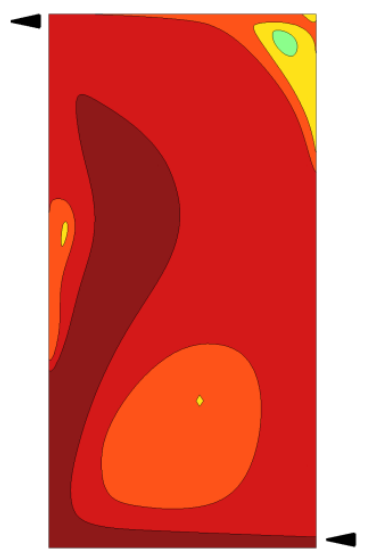

(b)

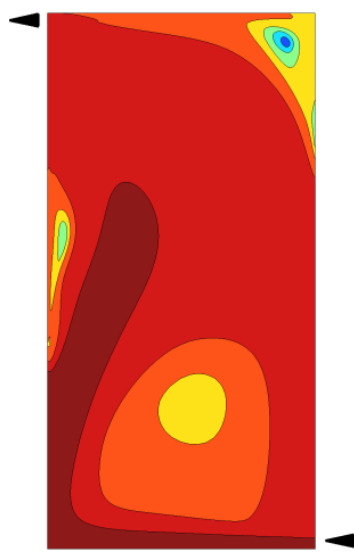

(c)

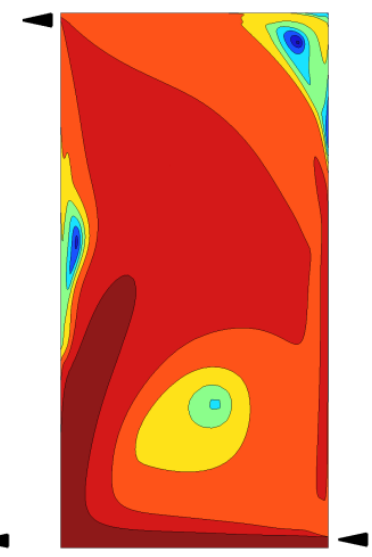

(d) 


\section{$\begin{array}{llllllll}50 & 54 & 58 & 62 & 66 & 70 & 74 & 78\end{array}$}

Figure 14. Temperature distribution in the feed side of the DCMD module for an inlet temperature of $80^{\circ} \mathrm{C}$ using membrane $\mathrm{M} 2$, (a) $2 \mathrm{~mm}$ above the membrane (top wall), (b) 1 $\mathrm{mm}$ above the membrane, (c) $0.5 \mathrm{~mm}$ above the membrane, and (d) $0.1 \mathrm{~mm}$ above the membrane.

Since temperatures at both sides of the membrane are part of the solution of the coupled approach, it is possible to post-process the temperature polarization coefficient $\left(\tau_{T}\right)$. The latter can be simply defined by:

$$
\tau_{T}=\frac{T_{m f}-T_{m p}}{T_{i n}-T_{p}}
$$

The definition of the temperature polarization coefficient in Eq. (29) means that the closer $T_{m f}-T_{m p}$ to $T_{i n}-T_{p}$ or equivalently as $\tau_{T}$ gets closer to one, the lesser the polarization.

The evolution of the average value of the polarization coefficient as a function of feed inlet temperature is represented in Figure 15. The polarization phenomenon, which affects the MD process, gets more severe for both membranes as the inlet temperature is increased, which is in accordance with previously reported investigations [44]. Overall, the average value behaves quasi-linearly for inlet temperatures ranging between $50^{\circ} \mathrm{C}$ and $80^{\circ} \mathrm{C}$.

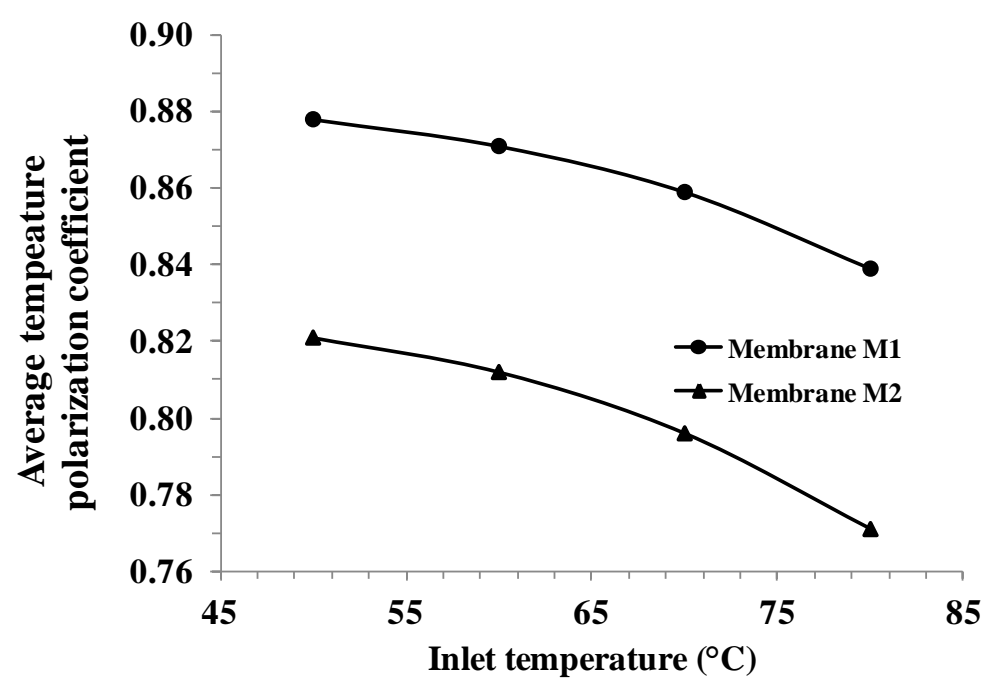


Figure 15. Evolution of the average temperature polarization coefficient as a function of feed inlet temperature.

The distribution of the temperature polarization coefficient over the membrane surface is represented in Figure 16 for both M1 and M2 membranes for the lowest and the highest inlet temperatures. The evolution of the coefficient exhibits the highest values at the inlet while the lowest values correspond to dead zones (left edge, top right corner of the top view). Therefore, the polarization phenomenon seems to be alleviated by high tangential velocities. Indeed, the large recirculation loop where an important velocity drop is observed near the feed inlet seems to promote polarization particularly for membrane M2. The lowest inlet temperatures seem to suffer less from polarization phenomenon which is in accordance with the fact that these are expected to produce smaller distillation rates.

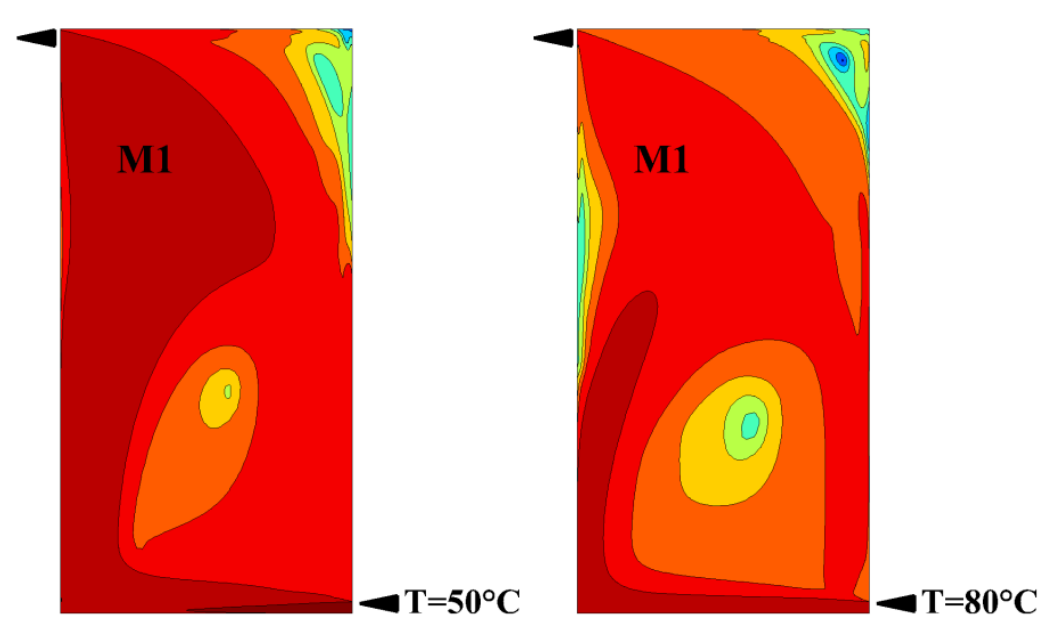

(a)
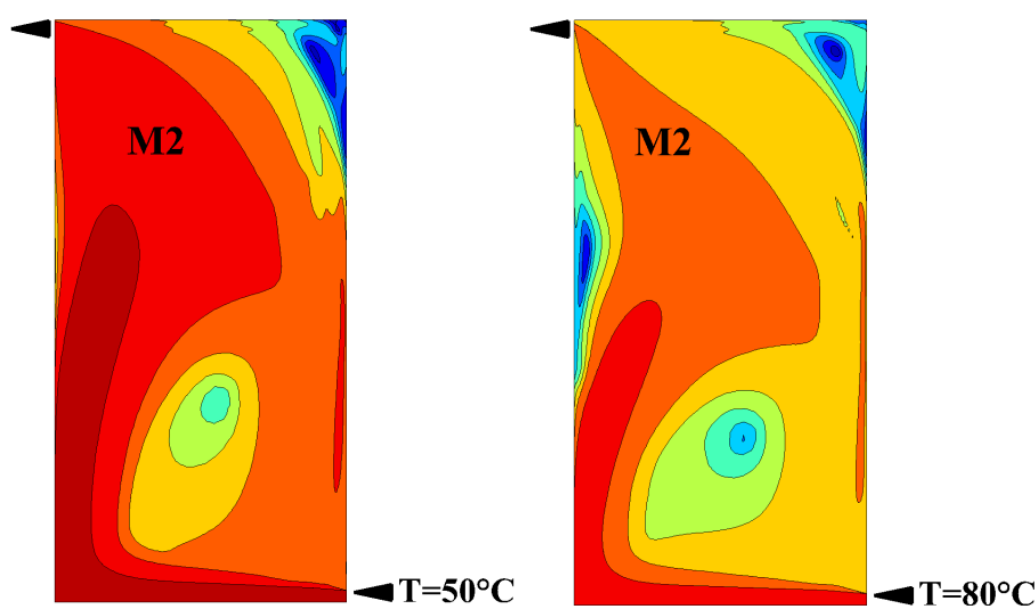

(b) 


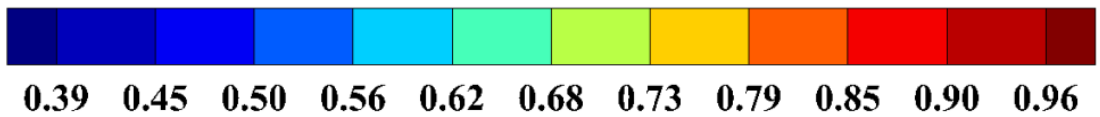

Figure 16. Distribution of the temperature polarization coefficient over the membrane surface for inlet temperatures of $50^{\circ} \mathrm{C}$ and $80^{\circ} \mathrm{C}$, (a) Membrane M1, and (b) Membrane

M2.

\subsection{Salinity distribution}

The advantage of the fully coupled approach is the ability to predict the distribution of the species mass fraction throughout the DCMD module. The mass fraction of $\mathrm{NaCl}$ species gives directly the value of salinity in the computational domain which enables an assessment of concentration polarization during the process. Salinity at the membrane surface is reported in Figure 17 for the two inlet temperatures of $50^{\circ} \mathrm{C}$ and $80^{\circ} \mathrm{C}$ for both membranes. For the lowest temperature, only slight changes are observed over the membrane surface. Indeed, for an inlet temperature of $50^{\circ} \mathrm{C}$, a difference of $2.3 \%$ and $3.5 \%$ occurs over the surface of membranes $\mathrm{M} 1$ and $\mathrm{M} 2$, respectively. However, for an inlet temperature of $80^{\circ} \mathrm{C}$ these differences increase to $7.4 \%$ and $10.4 \%$, respectively. Overall, it has to be noted that salinity distribution follows closely the flow pattern at higher temperatures. Regions with high salt concentration correspond to region of high water local depletion caused by relatively high vapor transfer rates. These regions exhibit the highest fluid velocities thus the thinnest temperature boundary layers. It is interesting to point out that tracking species individually using a multi-component diffusion approach can help improve DCMD module design. Indeed, the increase in salt concentration during the separation of concentrated solutions can ultimately lead to salt precipitation, therefore causing severe performance decrease.

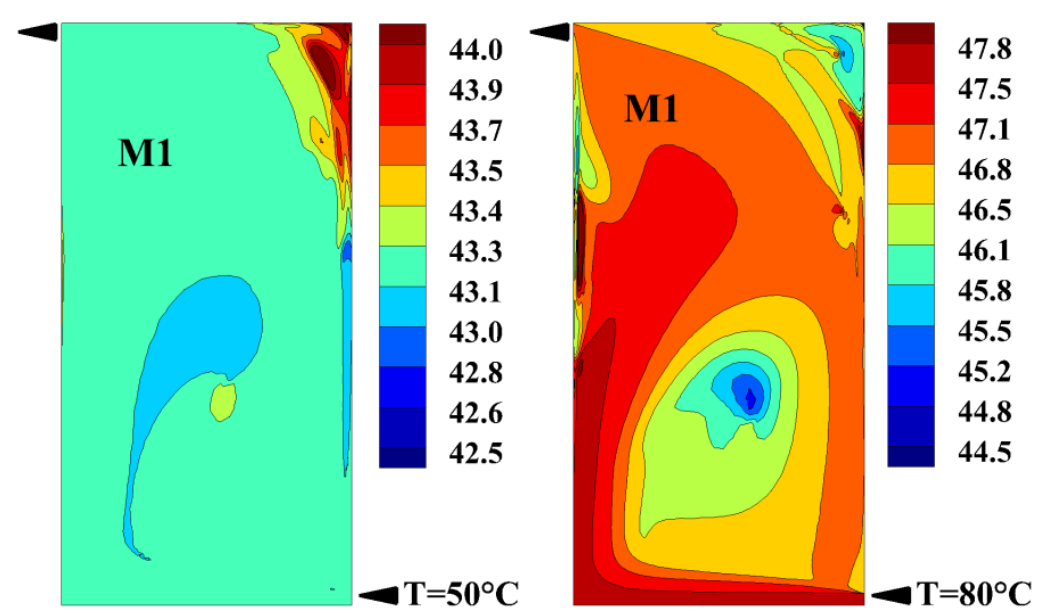


(a)

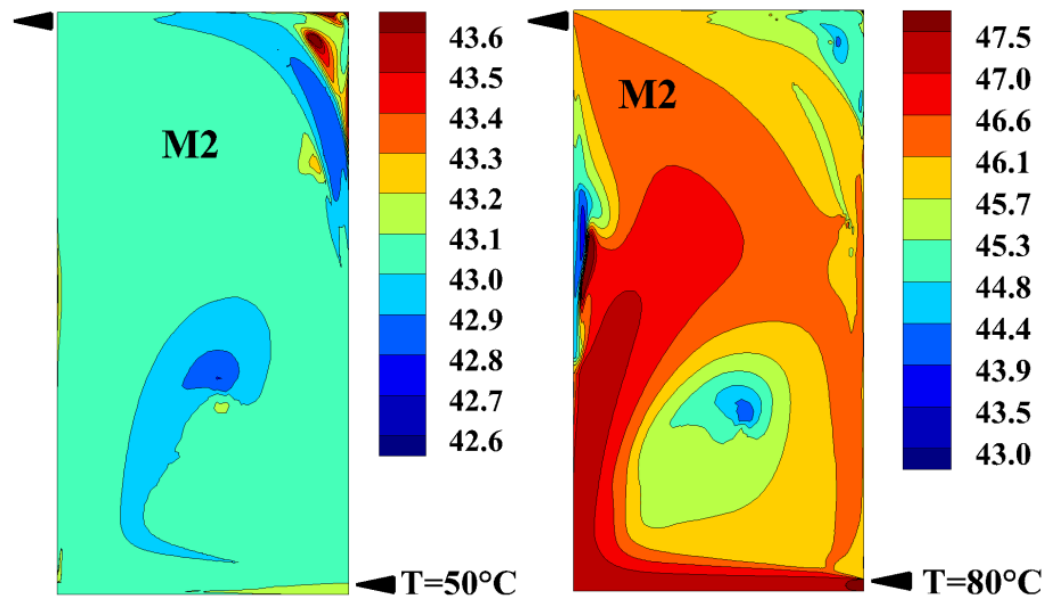

(b)

Figure 17. Distribution of salinity $\left(\mathrm{g} \cdot \mathrm{Kg}^{-1}\right)$ over the membrane surface for inlet temperatures of $50^{\circ} \mathrm{C}$ and $80^{\circ} \mathrm{C}$, (a) Membrane M1, and (b) Membrane M2.

The ratio of the inlet salinity $S_{i}$ over the salinity $S_{m}$ at the membrane surface can be used to assess the extent of concentration polarization, viz.

$$
\tau_{C}=\frac{S_{m}}{S_{i}}
$$

Following Eq. (30), the closer the coefficient to one, the lesser the concentration polarization.

The evolution of the average concentration polarization coefficient at the membrane surface as a function of feed temperature is represented in Figure 18. The average values are close to the maximum values observed in Figure 17. The average coefficient increases from approximately $3 \%$ at $50^{\circ} \mathrm{C}$ and exceeds $10 \%$ at $80^{\circ} \mathrm{C}$ and remains practically identical for both membranes. The lower the feed inlet temperature the less important the concentration polarization phenomenon which is in accordance with the fact that local salt species mass fraction is expected to increase at the membrane surface at higher inlet temperatures due to a increasing transfer rate of water species. 


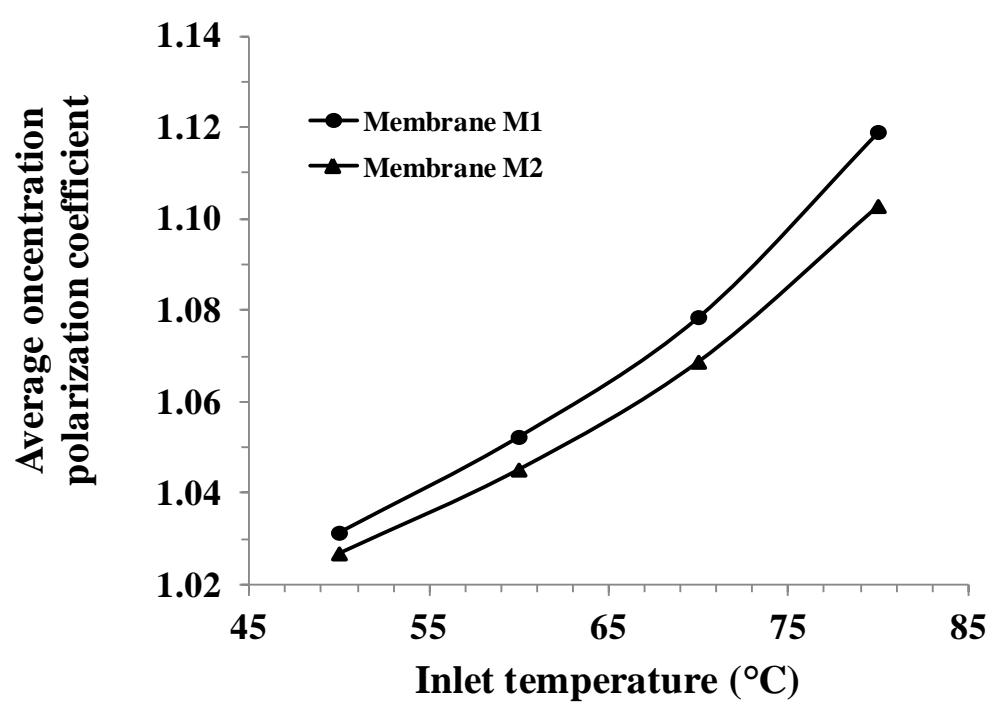

Figure 18. Evolution of the average concentation polarization coefficient at the membrane surface as a function of feed inlet temperature.

The distribution of the concentration polarization coefficient over the membrane surface is depicted in Figure 19. Analogously to the temperature polarization coefficient, the distribution is closely related to flow pattern. The coefficient variations over the surface show that concentration polarization is less important for the low feed inlet temperatures where the maximum values of the coefficient remain low (1.03), as they exhibit only a $3 \%$ shift from the value at the inlet. However, for the highest temperature used, the concentration polarization coefficient reaches $14 \%$ in regions of high permeate fluxes. Interestingly, for low temperatures, the concentration polarization is more important in dead zones where salt species seem to accumulate. However, unlike temperature polarization, at high temperatures the concentration polarization is more important in regions where high tangential velocities prevail due to higher water distillation rates. 

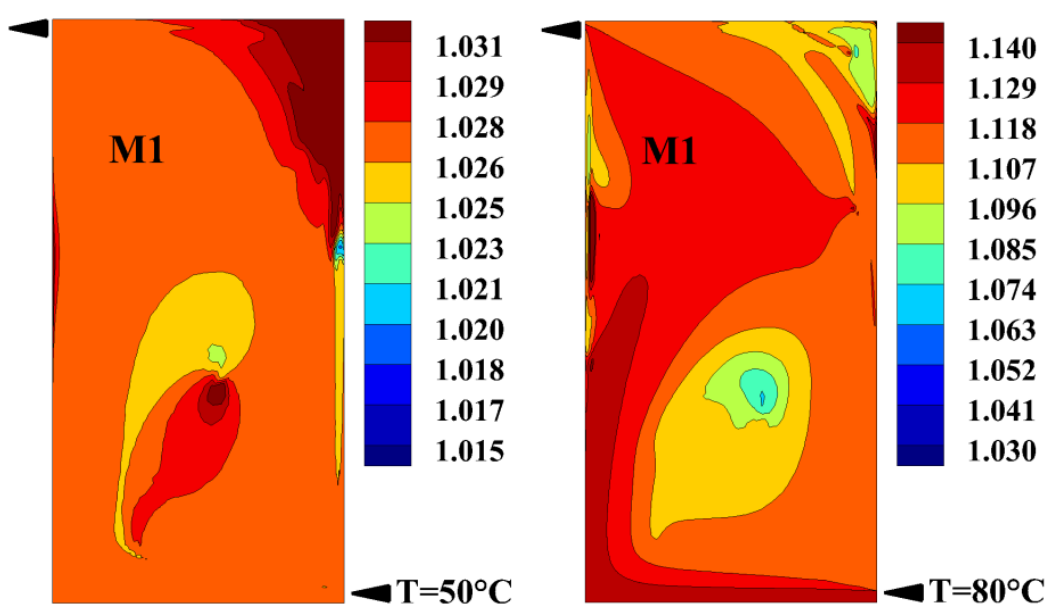

(a)
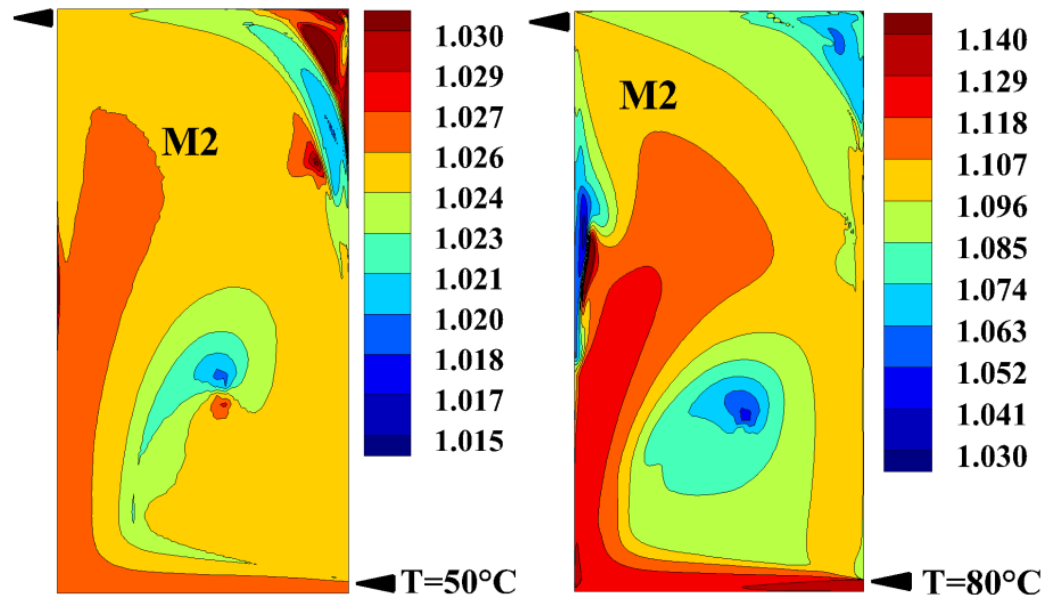

(b)

Figure 19. Distribution of the concentration polarization coefficient over the membrane surface for inlet temperatures of $50^{\circ} \mathrm{C}$ and $80^{\circ} \mathrm{C}$, (a) Membrane M1, and (b) Membrane M2.

\subsection{Permeate flux}

The permeate fluxes are predicted along with momentum, heat and mass transfer solution throughout the membrane channel. Upon convergence, the newly computed variables are printed out by the ADJUST_PROFILE UDF function, the procedure that has been used to update the heat transfer coefficient during computation (see flowchart presented in Figure 5). The permeate flux evolution at the membrane surface for both membranes M1 and M2 is given in Figure 20 for the lowest and highest inlet temperatures investigated. 

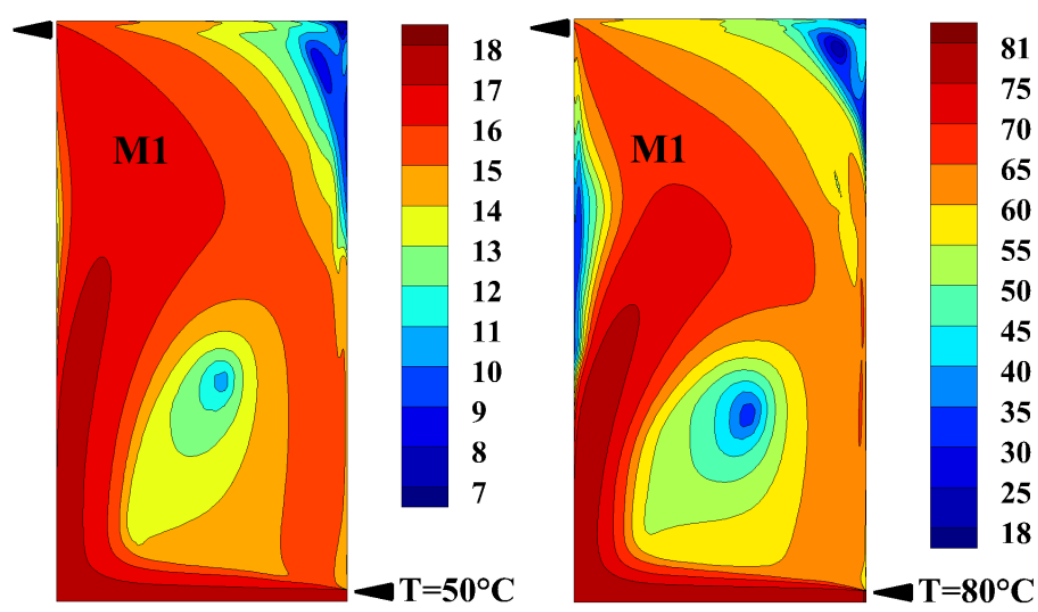

(a)
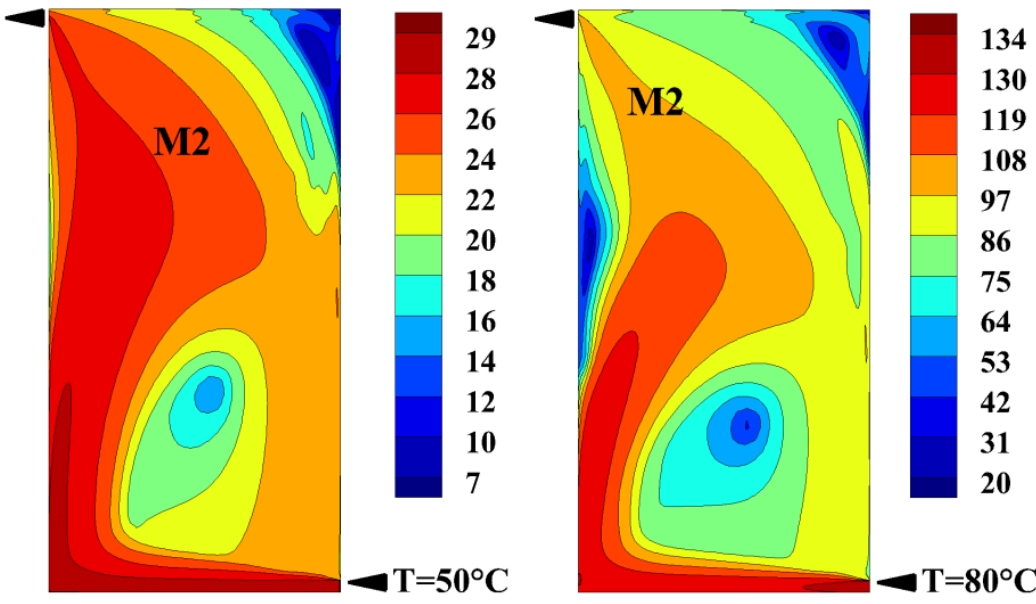

(b)

Figure 20. Distribution of permeate flux $\left(\mathrm{kg} \cdot \mathrm{m}^{-2} \cdot \mathrm{hr}^{-1}\right)$ over the membrane surface for inlet temperatures of $50^{\circ} \mathrm{C}$ and $80^{\circ} \mathrm{C}$, (a) Membrane M1, and (b) Membrane M2.

The distribution trend remains similar for both membranes with the highest flux values observed at the entrance where the highest temperature and velocities prevail. The flux remains relatively high along the inlet stream then toward the channel center and the module exit. However, a severe drop occurs at the dead zone of the top right corner of the channel for both membranes and both inlet temperatures while the recirculation loop at the edge seem to affect the permeate fluxes only at the highest temperature. An important permeate flux decrease is also observed at the center of the largest recirculation zone even though this region is close to the inlet. A zoom over the inlet area for an inlet temperature of $80^{\circ} \mathrm{C}$ using membrane M2 is shown in Figure 21. The permeate flux decreases significantly from $134 \mathrm{~kg} \cdot \mathrm{m}^{-2} \cdot \mathrm{hr}^{-1}$ to $40 \mathrm{~kg} \cdot \mathrm{m}^{-2} \cdot \mathrm{hr}^{-1}$ over a small distance of $3.0 \mathrm{~cm}$ (from the inlet to the center of 
the recirculation loop). This confirms that the presence of recirculation zones in MD modules can considerably affect their throughput, particularly for the highest temperatures. Overall, permeate fluxes follow closely the velocity distribution above the membrane surface (see Figure 13).

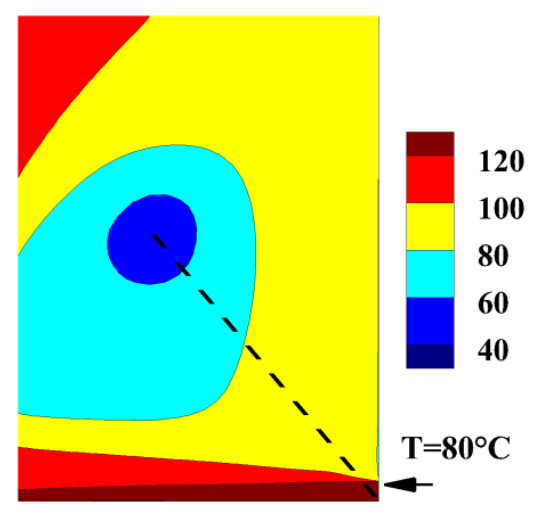

Figure 21. Permeate flux distribution $\left(\mathrm{kg} \cdot \mathrm{m}^{-2} \cdot \mathrm{hr}^{-1}\right)$ using membrane M2 for an inlet temperature of $80^{\circ} \mathrm{C}$ : zoom over the inlet zone.

The permeate flux distribution is in accordance with the distribution of salinity as well as the concentration polarization coefficient at the membrane surface depicted in Figures 17 and 19, respectively. Indeed, in regions of high fluxes, water consumption increases and mass transfer seems to limit water transport which results in higher "Salt" mass fraction. However, for the lowest temperatures, permeate fluxes remain low and "Salt" tend to accumulate in dead zones. It is understandable that concentration polarization is also more important for membrane M2 since higher permeate fluxes are produced using this membrane. Indeed, the higher polarization occurs in regions that are not sustained by high feed velocities. Nevertheless, these changes seem to have no impact on the calculated average values of the permeate flux, which agrees with reported investigations on concentration polarization [26]. Indeed, for instance for membrane $\mathrm{M} 2$ and inlet feed temperature of $80^{\circ} \mathrm{C}$, setting the consumption rate of water species at the membrane to zero results in less than $2 \%$ difference in the average value of the permeate flux. However, this low impact of concentration polarization could be related to the small size of the laboratory scale prototype. 


\section{Concluding remarks}

The effect of flow pattern on the distribution of permeate fluxes in DCMD has been investigated with numerical modeling. A coupled approach to simulate DCMD for desalination process was presented and simulations at the process scale were carried out using CFD software in a 3D domain of which boundary conditions are corrected by pore scale calculations. The implementation enables the simultaneous prediction of momentum, heat and species transport by combining process and pore scales without prior guess of heat or permeate fluxes across the domain boundaries. The numerical results, in terms of average permeate fluxes, are in very good agreement with experimental desalination data produced with two different membranes, with less than $6 \%$ error and no fitting parameter. The approach enabled to predict the distribution of permeate fluxes and salinity on the feed side as well as the temperature at both sides of the membrane. The temperature and concentration polarization coefficients were post-processed. Simulations carried out for the two different membranes show that flow pattern has an important effect on temperature and "Salt" species and consequently on the temperature and concentration polarization phenomena. Results confirmed that temperature polarization affects the DCMD process over the whole range of feed temperatures that were investigated; while concentration polarization could exceed $14 \%$ in regions of high permeate fluxes. The permeate fluxes were found to follow closely the fluid velocity distribution at the vicinity of the membrane. Indeed, since inlet velocities are tangential to the membrane surface, high permeate fluxes are sustained along the mainstream. However, the flux values are strongly affected by the presence of recirculation loops as well as dead zones. It was found that for such a relatively small prototype, the recirculation loop causes the flux to drop by a factor of 3 over very small regions. Moreover, for low operating temperatures, dead zones tend to accumulate salt. It is also very likely for these effects to become more severe if modules are scaled-up. Any geometry modification departing from the flat module, such as the inclusion of baffles should be carefully considered with the analysis of flow pattern. MD early module design should therefore focus mainly on flow pattern and avoid sudden changes in flow evolution as well as geometry cross section to avoid important recirculation loops and set proper exits location in order to minimize dead zones occurrence.

\section{Acknowledgments}

The research reported in this paper was supported by King Abdullah University of Science and Technology (KAUST), Saudi Arabia. 


\section{Nomenclature}

$a$ coefficient in Antoine's equation

$b$ coefficient in Antoine's equation

$c$ coefficient in Antoine's equation

$c_{p}$ specific heat at constant pressure

$c_{p v}$ vapor specific heat

$d_{h}$ hydraulic diameter

$h$ convective heat transfer coefficient

$h_{\text {ext }}$ external heat transfer coefficient

$k$ turbulent kinetic energy

$l$ characteristic length

$m_{w}$ weight of collected permeate

$p$ fluid pressure

$r$ pore radius

$s$ salinity

$t$ time interval

$u$ fluid velocity

$\bar{u}$ mean fluid velocity

$u^{\prime}$ fluid fluctuating velocity

$v_{i}$ fluid inlet velocity

$w$ species mass fraction

$z$ charge of salt ion

$A$ effective membrane area

$A_{c_{p}}$ coefficient in specific heat equation

$A_{\mu}$ coefficient in water viscosity equation

$B_{c_{p}}$ coefficient in specific heat equation

$B_{\mu}$ coefficient in water viscosity equation

$C$ membrane mass transfer coefficient 
$C_{1 \varepsilon}$ constant in $k-\varepsilon$ turbulence model

$C_{2 \varepsilon}$ constant in $k-\varepsilon$ turbulence model

$C_{c_{p}}$ coefficient in specific heat equation

$C_{\mu}$ constant in $k$ - $\varepsilon$ turbulence model

$D$ species diffusion coefficient in the feed

$D_{c_{p}}$ coefficient in specific heat equation

$D_{s}$ species diffusion coefficient in pores

$G_{k}$ generation of turbulent kinetic energy

$H$ fluid enthalpy

$H_{L}$ enthalpy of the permeate solution

$H_{v}$ vapor enthalpy

$J$ mass permeate flux

$J_{v}$ experimental water vapor flux

$M$ molecular weight

$\mathrm{Nu}$ Nusselt number

$P$ vapor pressure

$P_{a}$ air pressure inside pores

$P_{m f}$ vapor pressure at the feed side of the membrane

$P_{m p}$ vapor pressure at the permeate side of the membrane

$P_{s w}$ seawater vapor pressure

Pr Prandtl number

$\mathrm{Pr}_{\mathrm{t}}$ turbulent Prandtl number

$Q_{c}$ heat transferred by conduction across the membrane

$Q_{f}$ convective heat transferred from the feed to the membrane

$Q_{f}^{m}$ heat due to mass transfer from the feed to the membrane

$Q_{p}$ heat transferred from the membrane surface to the permeate side

$Q_{p}^{m}$ heat due to mass transfer from the membrane to the permeate side

$Q_{v}$ heat due to vapor transport across the membrane 
$R$ ideal gas constant

Re Reynolds number

$R_{m}$ membrane total resistance to heat transfer

$R_{p}$ permeate side resistance to heat transfer

$S$ modulus of mean rate of stress tensor

$\mathrm{Sc}_{\mathrm{t}}$ turbulent Schmidt number

$S_{i j}$ mean strain rate

$T$ temperature

$T_{a v}$ average temperature inside the pore

$T_{\text {ext }}$ permeate bulk temperature

$T_{f}$ feed temperature

$T_{\text {face }}$ temperature of mesh boundary face

$T_{i}$ fluid inlet temperature

$T_{m f}$ temperature at the membrane surface on the feed side

$T_{m p}$ temperature at the membrane surface on the permeate side

$T_{p}$ permeate temperature

$T_{w}$ boundary temperature

\section{Greek letters}

$\delta_{m}$ membrane thickness

$\delta_{i j}$ Kronecker delta

$\varepsilon$ turbulent kinetic energy dissipation

$\varepsilon_{m}$ membrane porosity

$\xi$ pore tortuosity

$\lambda_{g}$ thermal conductivity of the membrane fluid phase

$\lambda_{m}$ membrane composite thermal conductivity

$\lambda_{s}$ thermal conductivity of the membrane solid phase

$\lambda_{s w}$ thermal viscosity of seawater 
$\rho$ feed density

$\mu$ fluid viscosity

$\mu_{r}$ correction for seawater viscosity

$\mu_{s w}$ seawater viscosity

$\mu_{t}$ fluid turbulent viscosity

$\mu_{w}$ water viscosity

$\sigma_{\varepsilon}$ constant in $k-\varepsilon$ turbulence model

$\sigma_{k}$ constant in $k$ - $\varepsilon$ turbulence model

$\tau_{C}$ concentration polarization coefficient

$\tau_{T}$ temperature polarization coefficient

\section{Equation Section (Next)}

\section{Appendix A}

\section{Governing equations}

The continuity equation

$$
\frac{\partial \rho}{\partial t}+\frac{\partial}{\partial x_{i}}\left(\rho u_{i}\right)=0
$$

The momentum balance

$$
\frac{\partial}{\partial t}\left(\rho u_{i}\right)+\frac{\partial}{\partial x_{j}}\left(\rho u_{i} u_{j}\right)=-\frac{\partial p}{\partial x_{i}}+\frac{\partial}{\partial x_{j}}\left\{\mu\left(\frac{\partial u_{i}}{\partial x_{j}}+\frac{\partial u_{j}}{\partial x_{i}}-\frac{2}{3} \delta_{i j} \frac{\partial u_{l}}{\partial x_{l}}\right)\right\}+\frac{\partial}{\partial x_{j}}\left(-\rho \overline{u_{i}^{\prime} u_{j}^{\prime}}\right)
$$

where $u_{i}=\bar{u}_{i}+u_{i}^{\prime}$ is the sum of the mean and the fluctuating velocities while $-\rho \overline{u_{i}^{\prime} u_{j}^{\prime}}$ represents the Reynolds stress given by:

$$
-\rho \overline{u_{i}^{\prime} u_{j}^{\prime}}=\mu_{t}\left(\frac{\partial u_{i}}{\partial x_{j}}+\frac{\partial u_{j}}{\partial x_{i}}\right)-\frac{2}{3}\left(\rho k+\mu_{t} \frac{\partial u_{i}}{\partial x_{i}}\right) \delta_{i j}
$$

where $\mu_{t}$ represents the turbulent viscosity and $k$ the turbulent kinetic energy.

For incompressible flow with no buoyancy and no source terms, the transport equations for the turbulent kinetic energy and the dissipation rate are expressed as:

- Transport of turbulent kinetic energy 


$$
\frac{\partial}{\partial t}(\rho k)+\frac{\partial}{\partial x_{i}}\left(\rho k u_{i}\right)=\frac{\partial}{\partial x_{j}}\left\{\alpha \mu_{e f f} \frac{\partial k}{\partial x_{j}}\right\}+G_{k}-\rho \varepsilon
$$

- Transport of dissipation rate

$$
\frac{\partial}{\partial t}(\rho \varepsilon)+\frac{\partial}{\partial x_{i}}\left(\rho \varepsilon u_{i}\right)=\frac{\partial}{\partial x_{j}}\left\{\alpha \mu_{e f f} \frac{\partial \varepsilon}{\partial x_{j}}\right\}+C_{1 \varepsilon} \frac{\varepsilon}{k} G_{k}-C_{2 \varepsilon}^{*} \rho \frac{\varepsilon^{2}}{k}
$$

where $\mu_{\text {eff }}=\mu+\mu_{t}$ represents the effective turbulent viscosity and $\alpha$ an inverse effective Prandtl number estimated using the following equation:

$$
|\alpha-1.3929|^{0.6321}|\alpha+2.3929|^{0.3679}=1.333 \frac{\mu}{\mu_{e f f}}
$$

The effective viscosity is determined in the RNG model by solving a differential equation, viz.

$$
d\left(\frac{\rho^{2} k}{\sqrt{\varepsilon \mu}}\right)=1.72 \frac{\mu_{e f f} / \mu}{\sqrt{\left(\mu_{e f f} / \mu\right)^{3}-1+C_{v}}} d\left(\frac{\mu_{e f f}}{\mu}\right)
$$

The generation of turbulent kinetic energy due to mean velocity gradients, namely $G_{k}$ is related to the modulus of the mean rate of strain tensor $S$ by:

$$
G_{k}=\mu_{t} S^{2}
$$

where $S=\sqrt{2 S_{i j} S_{i j}}$ and the mean strain rate $S_{i j}=\frac{1}{2}\left(\frac{\partial u_{j}}{\partial x_{i}}+\frac{\partial u_{i}}{\partial x_{j}}\right)$.

The coefficient $C_{2 \varepsilon}^{*}$ of the last term of Eq. (A5) is given by:

$$
C_{2 \varepsilon}^{*}=C_{2 \varepsilon}+\frac{C_{\mu} \rho \eta^{3}\left(1-\eta / \eta_{0}\right)}{1+\beta \eta^{3}}
$$

with $\eta=S k / \varepsilon$.

The model constants in Eq. (A5), Eq. (A7) and Eq. (A9) are the ANSYS fluent preset values $C_{1 \varepsilon}=1.42, C_{2 \varepsilon}=1.68, C_{v}=100, \eta_{0}=4.38, \beta=0.012$ and $C_{\mu}=0.0845$.

\section{Heat balance}

Convective heat transfer with no viscous heating and no source term is considered which is governed by:

$$
\frac{\partial(\rho H)}{\partial t}+\frac{\partial}{\partial x_{i}}\left[u_{i}(\rho H)\right]=\frac{\partial}{\partial x_{j}}\left[\left(\lambda+\frac{c_{p} \mu_{t}}{\operatorname{Pr}_{\mathrm{t}}}\right) \frac{\partial T}{\partial x_{j}}-\sum_{k} H^{(k)} J^{(k)}\right]
$$


with $\operatorname{Pr}_{t}$ the turbulent Prandtl number, $c_{p}$ the specific heat at constant pressure, $J^{(k)}$ the diffusion flux of species " $k$ ", $H^{(k)}$ the enthalpy of species " $k$ " and $H$ the total enthalpy given by:

$$
H=\sum_{k} w^{(k)} \int_{298.15}^{T} c_{p}^{(k)} d T
$$

where $w^{(k)}$ is the mass fraction of species " $k$ " and $c_{p}^{(k)}$ the specific heat at constant pressure of species " $k$ ".

\section{Mass balance}

Regarding mass transfer calculations, seawater is considered as a mixture of $\mathrm{H}_{2} \mathrm{O}$ and $\mathrm{NaCl}$ species. Species transport is modeled using a single phase multi-component approach with no reaction which is governed by the following conservation form for each species " $k$ ",

$$
\frac{\partial\left(\rho w^{(k)}\right)}{\partial t}+\frac{\partial}{\partial x_{i}}\left(\rho u_{i} w^{(k)}\right)=-\frac{\partial J_{i}^{(k)}}{\partial x_{i}}
$$

With no Soret effect, the diffusion flux is given by:

$$
J_{i}^{(k)}=-\left(\rho D^{(k)}+\frac{\mu_{t}}{\mathrm{Sc}_{\mathrm{t}}}\right) \frac{\partial w^{(k)}}{\partial x_{i}}
$$

where $D^{(k)}$ is the diffusion coefficient of species " $k$ " and $\mathrm{Sc}_{\mathrm{t}}$ the turbulent Schmidt number while the turbulent viscosity $\mu_{t}$ is determined via the effective viscosity by solving Eq. (A7).

The rate of water consumption at the membrane surface due to the distillation process is determined by:

$$
\rho D^{(k)} \frac{\partial w^{(k)}}{\partial \mathrm{n}}=J
$$

where $J$ is the permeate mass flux.

\section{References}

[1] H.Y. Wu, M. Tay, R.W. Field, Novel method for the design and assessment of direct contact membrane distillation modules, Journal of Membrane Science, 513 (2016) 260-269.

[2] D. Winter, J. Koschikowski, F. Gross, D. Maucher, D. Düver, M. Jositz, T. Mann, A. Hagedorn, Comparative analysis of full-scale membrane distillation contactors - methods and modules, Journal of Membrane Science, 524 (2017) 758-771. 
[3] C.-D. Ho, H. Chang, C.-H. Tsai, P.-H. Lin, Theoretical and Experimental Studies of a Compact Multiunit Direct Contact Membrane Distillation Module, Industrial \& Engineering Chemistry Research, 55 (2016) 5385-5394.

[4] G. Dong, J.F. Kim, J.H. Kim, E. Drioli, Y.M. Lee, Open-source predictive simulators for scale-up of direct contact membrane distillation modules for seawater desalination, Desalination, 402 (2017) $72-87$.

[5] A. Criscuoli, Improvement of the Membrane Distillation performance through the integration of different configurations, Chemical Engineering Research and Design, 111 (2016) 316-322.

[6] S. Al-Sharif, M. Albeirutty, A. Cipollina, G. Micale, Modelling flow and heat transfer in spacerfilled membrane distillation channels using open source CFD code, Desalination, 311 (2013) 103-112.

[7] M. Shakaib, S.M.F. Hasani, I. Ahmed, R.M. Yunus, A CFD study on the effect of spacer orientation on temperature polarization in membrane distillation modules, Desalination, 284 (2012) 332-340.

[8] M. Shakaib, S.M.F. Hasani, M.E.-u. Haque, I. Ahmed, R.M. Yunus, A CFD study of heat transfer through spacer channels of membrane distillation modules, Desalination and Water Treatment, 51 (2013) 3662-3674.

[9] H. Yu, X. Yang, R. Wang, A.G. Fane, Analysis of heat and mass transfer by CFD for performance enhancement in direct contact membrane distillation, Journal of Membrane Science, 405-406 (2012) $38-47$.

[10] A. Cipollina, A. Di Miceli, J. Koschikowski, G. Micale, L. Rizzuti, CFD simulation of a membrane distillation module channel, Desalin. Water Treat., 6 (2009) 177-183.

[11] A. Cipollina, G. Micale, L. Rizzuti, Membrane distillation heat transfer enhancement by CFD analysis of internal module geometry, Desalin. Water Treat., 25 (2011) 195-209.

[12] H. Hayer, O. Bakhtiari, T. Mohammadi, Simulation of momentum, heat and mass transfer in direct contact membrane distillation: A computational fluid dynamics approach, J. Ind. Eng. Chem., 21 (2015) 1379-1382.

[13] F.N. Ponzio, A. Tamburini, A. Cipollina, G. Micale, M. Ciofalo, Experimental and computational investigation of heat transfer in channels filled by woven spacers, Int. J. Heat Mass Transf., 104 (2017) 163-177.

[14] S. Upadhyaya, K. Singh, S.P. Chaurasia, R.K. Dohare, M. Agarwal, Mathematical and CFD modeling of vacuum membrane distillation for desalination, Desalin. Water Treat., 57 (2016) 1195611971.

[15] B. Lian, Y. Wang, P. Le-Clech, V. Chen, G. Leslie, A numerical approach to module design for crossflow vacuum membrane distillation systems, J. Membr. Sci., 510 (2016) 489-496.

[16] H. Ahadi, J. Karimi-Sabet, M. Shariaty-Niassar, The Effect of Module Geometry on Heat and Mass Transfer in Membrane Distillation, Chem. Prod. Process Model., 11 (2016) 35-39.

[17] C. Katsandri, M.M. Vahdati, A theoretical and experimental method of enhancing mass transfer in flat plate membrane distillation using CFD, 05AIChE: 2005 AIChE Annual Meeting and Fall Showcase, American Institute of Chemical Engineers, Cincinnati, OH, 2005.

[18] H. Yu, X. Yang, R. Wang, A.G. Fane, Numerical simulation of heat and mass transfer in direct membrane distillation in a hollow fiber module with laminar flow, J. Membr. Sci., 384 (2011) 107 116.

[19] I. Janajreh, D. Suwwan, Numerical Simulation of Direct Contact Membrane Desalination, IAJCISAM International ConferenceOrlando, Florida, 2014. 
[20] I. Janajreh, D. Suwwan, R. Hashaikeh, Theoretical and experimental study of direct contact membrane distillation, Desalin. Water Treat., 57 (2016) 15660-15675.

[21] A. Katsandri, A theoretical analysis of a spacer filled flat plate membrane distillation modules using CFD: Part I: Velocity and shear stress analysis, Desalination, (2015).

[22] H. Chang, J.A. Hsu, C.L. Chang, C.D. Ho, CFD Study of Heat Transfer Enhanced Membrane Distillation Using Spacer-Filled Channels, in: J. Yan, T. Shamim, S.K. Chou, H. Li (Eds.) 7th International Conference on Applied Energy, ICAE 2015, Elsevier Ltd, 2015, pp. 3213-3219.

[23] H. Chang, J.A. Hsu, C.L. Chang, C.D. Ho, CFD Simulation of Direct Contact Membrane Distillation Modules with Rough Surface Channels, in: J. Yan, T. Shamim, S.K. Chou, H. Li (Eds.) 7th International Conference on Applied Energy, ICAE 2015, Elsevier Ltd, 2015, pp. 3083-3090.

[24] H. Chang, C.D. Ho, J.A. Hsu, Analysis of heat transfer coefficients in direct contact membrane distillation modules using CFD simulation, J. Appl. Sci. Eng., 19 (2016) 197-206.

[25] M. Hasanizadeh, P. Jafari, B. Farshighazani, M.K. Moraveji, CFD simulation of heat and mass transport for water transfer through hydrophilic membrane in direct-contact membrane distillation process, Desalin. Water Treat., 57 (2016) 18109-18119.

[26] M.M.A. Shirazi, A. Kargari, A.F. Ismail, T. Matsuura, Computational Fluid Dynamic (CFD) opportunities applied to the membrane distillation process: State-of-the-art and perspectives, Desalination, 377 (2016) 73-90.

[27] ANSYS-fluent, ANSYS, Inc.

[28] A.S. Alsaadi, N. Ghaffour, J.D. Li, S. Gray, L. Francis, H. Maab, G.L. Amy, Modeling of air-gap membrane distillation process: A theoretical and experimental study, J. Membr. Sci., 445 (2013) 5365 .

[29] A. Alkhudhiri, N. Darwish, N. Hilal, Membrane distillation: A comprehensive review, Desalination, 287 (2012) 2-18.

[30] E. Curcio, E. Drioli, Membrane Distillation and Related Operations-A Review, Separation \& Purification Reviews, 34 (2005) 35-86.

[31] L. Francis, N. Ghaffour, A.S. Alsaadi, S.P. Nunes, G.L. Amy, Performance evaluation of the DCMD desalination process under bench scale and large scale module operating conditions, Journal of Membrane Science, 455 (2014) 103-112.

[32] M. Khayet, Membranes and theoretical modeling of membrane distillation: A review, Advances in Colloid and Interface Science, 164 (2011) 56-88.

[33] J.-G. Lee, Y.-D. Kim, W.-S. Kim, L. Francis, G. Amy, N. Ghaffour, Performance modeling of direct contact membrane distillation (DCMD) seawater desalination process using a commercial composite membrane, Journal of Membrane Science, 478 (2015) 85-95.

[34] A.S. Alsaadi, L. Francis, G.L. Amy, N. Ghaffour, Experimental and theoretical analyses of temperature polarization effect in vacuum membrane distillation, Journal of Membrane Science, 471 (2014) 138-148.

[35] I. Hitsov, T. Maere, K. De Sitter, C. Dotremont, I. Nopens, Modelling approaches in membrane distillation: A critical review, Sep. Purif. Technol., 142 (2015) 48-64.

[36] M. Khayet, T. Matsuura, Membrane Distillation Principles, Elsevier, Amsterdam, The Netherlands, 2011.

[37] J. Phattaranawik, R. Jiraratananon, A.G. Fane, Effect of pore size distribution and air flux on mass transport in direct contact membrane distillation, J. Membr. Sci., 215 (2003) 75-85. 
[38] M. Khayet, A. Velasquez, J.I. Mengual, Modelling mass transport through a porous partition: Effect of pore size distribution, Journal of Non-Equilibrium Thermodynamics, 29 (2005) 279-299.

[39] M. Qtaishat, T. Matsuura, B. Kruczek, M. Khayet, Heat and mass transfer analysis in direct contact membrane distillation, Desalination, 219 (2008) 272-292.

[40] J. Phattaranawik, R. Jiraratananon, Direct contact membrane distillation: effect of mass transfer on heat transfer, Journal of Membrane Science, 188 (2001) 137-143.

[41] K.W. Lawson, D.R. Lloyd, Membrane distillation. II. Direct contact MD, Journal of Membrane Science, 120 (1996) 123-133.

[42] J.M. Smith, H.C. Van Ness, M.M. Abbott, Introduction to Chemical Engineering Thermodynamics, fifth edition ed., Mc Graw Hill, New York, 1996.

[43] M.N. Ozisik, Heat Transfer: A Basic Approach, McGraw-Hill, New York, 1985.

[44] J. Phattaranawik, R. Jiraratananon, A.G. Fane, Heat transport and membrane distillation coefficients in direct contact membrane distillation, J. Membr. Sci., 212 (2003) 177-193.

[45] L. Francis, N. Ghaffour, A.A. Alsaadi, G.L. Amy, Material gap membrane distillation: A new design for water vapor flux enhancement, Journal of Membrane Science, 448 (2013) 240-247.

[46] M. Matyka, A. Khalili, Z. Koza, Tortuosity-porosity relation in porous media flow, Physical Review E, 78 (2008) 026306.

[47] J.A. Dean, Lange's handbook of chemistry, 15 ed., McGraw-Hill1999.

[48] M.H. Sharqawy, J.H. Lienhard, S.M. Zubair, Thermophysical properties of seawater: a review of existing correlations and data, Desalination and Water Treatment, 16 (2010) 354-380.

[49] D. Caldwell, Thermal conductivity of sea water, Deep Sea Research and Oceanographic Abstracts, 21 (1974) 131-137.

[50] CRC Handbook of Chemistry and Physics, 84th Edition ed., CRC Press2003-2004.

[51] B.R. Munson, T.H. Okiishi, W.W. Huebsch, A.P. Rothmayer, Fundamentals of Fluid Mechanics, 7th edition ed., John Wiley and Sons, Inc., USA, 2013.

[52] C.-J. Chen, S.-Y. Jaw, Fundamentals of Turbulence Modeling, Taylor and Francis1998.

[53] A. Neale, D. Derome, B. B., J. Carmeliet, Determination of surface convective heat transfer coefficients by CFD, 11th Canadian Conference on Building Science and TechnologyBanff, Alberta, Canada, 2007.

[54] M.A.R. Sharif, K.K. Mothe, Evaluation of Turbulence Models in the Prediction of Heat Transfer Due to Slot Jet Impingement on Plane and Concave Surfaces, Numerical Heat Transfer, Part B: Fundamentals, 55 (2009) 273-294. 